\title{
TWO-DIMENSIONAL BUMPS IN PIECEWISE SMOOTH NEURAL FIELDS WITH SYNAPTIC DEPRESSION*
}

\author{
PAUL C. BRESSLOFF ${ }^{\dagger}$ AND ZACHARY P. KILPATRICK ${ }^{\ddagger}$
}

\begin{abstract}
We analyze radially symmetric bumps in a two-dimensional piecewise-smooth neural field model with synaptic depression. The continuum dynamics is described in terms of a nonlocal integrodifferential equation, in which the integral kernel represents the spatial distribution of synaptic weights between populations of neurons whose mean firing rate is taken to be a Heaviside function of local activity. Synaptic depression dynamically reduces the strength of synaptic weights in response to increases in activity. We show that in the case of a Mexican hat weight distribution, sufficiently strong synaptic depression can destabilize a stationary bump solution that would be stable in the absence of depression. Numerically it is found that the resulting instability leads to the formation of a traveling spot. The local stability of a bump is determined by solutions to a system of pseudolinear equations that take into account the sign of perturbations around the circular bump boundary.
\end{abstract}

Key words. neural fields, synaptic depression, piecewise-smooth dynamics

AMS subject classification. $92 \mathrm{C} 20$

DOI. $10.1137 / 100799423$

1. Introduction. Continuum neural field models provide an important example of spatially extended excitable systems with nonlocal interactions. These models represent the large-scale dynamics of populations of neurons in terms of nonlinear integrodifferential equations, whose associated integral kernels represent the spatial distribution of neuronal synaptic connections $[2,3,5,11,40]$. As in the case of nonlinear PDE models of diffusively coupled excitable systems [17], neural field models can exhibit a variety of coherent pulse-like structures, including both stationary and traveling solitary pulses. Traveling pulses tend to occur when synaptic connections are predominantly excitatory and there is some form of slow local adaptation or recovery [30], whereas stationary pulses (activity bumps) occur in the presence of lateral inhibition $[2,31]$. The formation of localized activity states can be used to model a number of neurobiological phenomena. For example, traveling pulses have been observed in disinhibited slice preparations $[6,41,42]$ using voltage sensitive dyes and multiple electrodes. A second example is given by a delayed response task in which an animal is required to retain information of a sensory cue across a delay period between the stimulus and behavioral response. Physiological recordings in the prefrontal cortex have shown that spatially localized groups of neurons fire during the recall task and then stop firing once the task has finished [38]. Thus persistent localized states of activity are thought to be neural correlates of spatial working memory.

*Received by the editors June 18, 2010; accepted for publication (in revised form) December 7, 2010; published electronically March 3, 2011. This publication was based on work supported in part by the National Science Foundation (DMS-0813677) and by award KUK-C1-013-4 made by King Abdullah University of Science and Technology (KAUST).

http://www.siam.org/journals/siap/71-2/79942.html

$\dagger$ Mathematical Institute, University of Oxford, 24-29 St. Giles', Oxford OX1 3LB, UK, and Department of Mathematics, University of Utah, Salt Lake City, UT 84112 (bressloff@maths.ox.ac.uk). The work of this author was partially supported by the Royal Society Wolfson Foundation.

${ }^{\ddagger}$ Department of Mathematics, University of Utah, Salt Lake City, UT 84112 (kilpatri@math.utah. edu). 
The simplest example of a one-dimensional (1D) neural field model is the scalar equation [2]

$$
\tau \frac{\partial u(x, t)}{\partial t}=-u(x, t)+\int_{-\infty}^{\infty} w\left(x-x^{\prime}\right) f\left(u\left(x^{\prime}, t\right)\right) \mathrm{d} x^{\prime}+h(x, t) .
$$

The neural field $u(x, t)$ represents the local activity of a population of neurons at position $x$ at time $t, \tau$ is a membrane or synaptic time constant, $h(x, t)$ represents an external input, and $w(x)$ is a synaptic weight distribution. We assume that $w$ is a continuous function satisfying $w(-x)=w(x)$ and $\int_{-\infty}^{\infty} w(x) \mathrm{d} x<\infty$. The nonlinearity $f$ denotes an output firing rate function. A typical choice for $f$ is a bounded, positive monotonic function such as the sigmoid

$$
f(u)=\frac{1}{1+\mathrm{e}^{-\eta(u-\theta)}},
$$

with gain $\eta$ and threshold $\theta$. Considerable insight into particular classes of spatially localized solutions of (1.1) and various generalizations involving vector-valued fields can be obtained by taking the high-gain limit $\eta \rightarrow \infty$ such that $f$ becomes a Heaviside function

$$
f(u)=H(u-\theta)= \begin{cases}0 & \text { if } u<\theta \\ 1 & \text { if } u>\theta\end{cases}
$$

It is then possible to establish existence of stationary and traveling pulse solutions by explicit construction, and to determine local stability in terms of an associated Evans function by linearizing the neural field equations about the pulse solution $[2$, $7,30,31,32,43]$. In the case of stationary pulses or bumps, local stability reduces to the problem of calculating the effects of perturbations at the bump boundary, where $u(x)=\theta$.

Equation (1.1) was first analyzed in detail by Amari [2], who showed that in the case of a Heaviside function $H$ and a homogeneous external input $h$, the network can support a stable stationary bump solution when the weight distribution $w(x)$ is given by a so-called Mexican hat function with the following properties: ${ }^{1}$

(i) $w(x)>0$ for $x \in\left[0, x_{0}\right)$ with $w\left(x_{0}\right)=0$.

(ii) $w(x)<0$ for $x \in\left(x_{0}, \infty\right)$.

(iii) $w(x)$ is decreasing on $\left[0, x_{0}\right]$.

(iv) $w(x)$ has a unique minimum on $\mathbb{R}^{+}$at $x=x_{1}$ with $x_{1}>x_{0}$ and $w(x)$ strictly increasing on $\left(x_{1}, \infty\right)$.

On the other hand, in the case of a purely excitatory network with $w(x)$ a positive, monotonically decreasing function, any bump solution is unstable and tends to break up into a pair of counterpropagating fronts. Following Amari's original analysis, Kishimoto and Amari [23] proved the existence of a stationary pulse for a smooth

\footnotetext{
${ }^{1}$ The Mexican hat weight function is based on the assumption that there is short-range excitation and long-range inhibition. From a mathematical perspective, it would also be possible to generate stable bumps using an inverted Mexican hat function. Which form is more realistic from the biological perspective depends on which classes of neurons are being taken into account by the neural field model. For example, in the visual cortex it is known that excitatory pyramidal cells make both local synaptic contacts as well as long-range horizontal connections. However, the latter innervate both excitatory and local inhibitory neurons so they could have a net inhibitory effect, thus providing a possible source of long-range inhibition; whether long-range connections generate net excitation or net inhibition also depends on stimulus conditions [27].
} 
sigmoidal nonlinearity $f$, rather than a Heaviside function, using a fixed point theorem. Moreover, rigorous functional analytical techniques have been used to study the existence and (absolute) stability of stationary bump solutions for a general class of neural field models with smooth $f$, where the spatial domain is taken to be bounded rather than infinite [12].

The constructive approach based on the use of Heaviside functions has been generalized in a number of ways (see the review by Coombes [5]). These include more general weight distributions for which multiple bump states can arise [24, 25], inhomogeneous neural fields [18], two-dimensional (2D) bumps [14, 15, 25, 39], and weakly interacting bumps [4]. There has also been a lot of recent interest in neural field models with some form of local negative feedback. The inclusion of negative feedback is motivated by the fact that the scalar model given by (1.1) cannot support traveling pulse solutions in the absence of synaptic inhibition, which is inconsistent with what is observed in disinhibited slice experiments $[6,41,42]$. Negative feedback is typically taken to be linear by analogy with the FitzHugh-Nagumo equation [13], leading to a neural field model of the form $[30,31]$

$$
\begin{aligned}
\tau \frac{\partial u(x, t)}{\partial t} & =-u(x, t)+\int_{-\infty}^{\infty} w\left(x, x^{\prime}\right) f\left(u\left(x^{\prime}, t\right)\right) \mathrm{d} x^{\prime}-v(x, t)+h(x, t), \\
\tau_{v} \frac{\partial v(x, t)}{\partial t} & =\gamma u(x, t)-v(x, t) .
\end{aligned}
$$

The auxiliary field $v(x, t)$ represents a slow local negative feedback component with $\tau_{v}$ and $\gamma$ determining the time constant and strength of the feedback, respectively. One of the interesting dynamical consequences of linear negative feedback is that the linear operator obtained by linearizing about a stationary bump solution can have complex-valued eigenvalues, thus providing a possible mechanism for the generation of spatially structured oscillations [14, 15, 33, 35].

One possible limitation of linear negative feedback is that it does not have a direct physiological interpretation. This motivates the study of neural field models with more realistic nonlinear forms of negative feedback such as synaptic depression. Synaptic depression is the process by which presynaptic resources such as chemical neurotransmitters or synaptic vesicles are depleted [45]. It can be incorporated into the scalar neural field model (1.1) by introducing a dynamic prefactor $q$ in the nonlocal term according to $[19,20]$

$$
\begin{aligned}
\tau \frac{\partial u(x, t)}{\partial t} & =-u(x, t)+\int_{-\infty}^{\infty} q\left(x^{\prime}, t\right) w\left(x-x^{\prime}\right) f\left(u\left(x^{\prime}, t\right)\right) \mathrm{d} x^{\prime}, \\
\frac{\partial q(x, t)}{\partial t} & =\frac{1-q(x, t)}{\alpha}-\beta q(x, t) f(u(x, t)) .
\end{aligned}
$$

The factor $q(x, t)$ can be interpreted as a measure of the fraction of available presynaptic resources, which are depleted at a rate $\beta f[34,37]$ and are recovered on a timescale specified by the constant $\alpha$ (experimentally shown to be $200-800 \mathrm{msec}[1,36,37]$ ). Suppose that the strength of a synapse is reduced by a factor $\kappa$ of its maximal value in response to a sustained input of rate $f=1$ with $\kappa \sim 0.05-0.9$ [1]. A simple steady-state calculation shows that $\beta \approx(1-\kappa) /(\kappa \alpha)$. Hence $\beta \sim 0.0001-0.1(\mathrm{~ms})^{-1}$ for the given range of values of $\alpha$. In previous work we focused on the role of synaptic depression in generating spatially structured oscillations in 1D [19] and 2D [20] excitatory neural networks with continuous firing rate functions. In the high-gain limit, oscillatory solutions no longer exist but stationary and traveling wave solutions 
can be constructed. However, the presence of a Heaviside function in the dynamics of the synaptic depression variable means that the resulting dynamical system is piecewise smooth, which considerably complicates the stability analysis. Indeed, we have recently shown how the local stability of a 1D stationary bump can be formulated in terms of a system of pseudolinear equations that keep track of the sign of perturbations of the bump boundary [21]. Solutions to these equations establish that sufficiently strong synaptic depression can destabilize a bump. In this paper we extend our analysis of piecewise smooth neural fields with synaptic depression to the case of a 2D network. We also give a more detailed derivation of our previous stability results for a $1 \mathrm{D}$ network.

\section{One-dimensional bumps.}

2.1. Existence of one-dimensional bumps. On setting $f(u)=H(u-\theta)$, a stationary solution $(U(x), Q(x))$ of (1.6) satisfies the pair of equations

$$
\begin{aligned}
& U(x)=\int_{-\infty}^{\infty} Q\left(x^{\prime}\right) w\left(x-x^{\prime}\right) H\left(U\left(x^{\prime}\right)-\theta\right) \mathrm{d} x^{\prime}, \\
& Q(x)=1-\frac{\alpha \beta}{1+\alpha \beta} H(U(x)-\theta) .
\end{aligned}
$$

Let $R[U]=\{x \mid U(x)>\theta\}$ be the region over which the field is excited or superthreshold. Exploiting the fact that any solution can be arbitrarily translated along the $x$-axis, we define a stationary bump solution of half-width $a$ to be one for which $R[U]=(-a, a)$. Substituting (2.2) into (2.1) then yields

$$
U(x)=\frac{1}{1+\alpha \beta}[W(x+a)+W(x-a)],
$$

where $W(x)=\int_{0}^{x} w(y) d y$. As a simple example, consider a Mexican hat distribution given by the difference-of-exponentials

$$
w\left(\left|x-x^{\prime}\right|\right)=\mathrm{e}^{-\left|x-x^{\prime}\right|}-A \mathrm{e}^{-\left|x-x^{\prime}\right| / \sigma} .
$$

Substituting the weight function (2.4) into the steady-state solution for $U(x)$ and evaluating the integral yields

$$
U(x)=\frac{1}{(1+\alpha \beta)}\left\{\begin{array}{cl}
2 \sinh a \mathrm{e}^{-x}-2 A \sigma \sinh (a / \sigma) \mathrm{e}^{-x / \sigma}, & x>a, \\
2-2 \mathrm{e}^{-a} \cosh x-2 A \sigma\left[1-\mathrm{e}^{-a / \sigma} \cosh (x / \sigma)\right], & |x|<a, \\
2 \sinh a \mathrm{e}^{x}-2 A \sigma \sinh (a / \sigma) \mathrm{e}^{x / \sigma}, & x<-a .
\end{array}\right.
$$

Applying the threshold conditions $U( \pm a)=\theta$, we arrive at an implicit expression relating the bump half-width $a$ to all other parameters: ${ }^{2}$

$$
\frac{1}{(1+\alpha \beta)}\left[1-\mathrm{e}^{-2 a}-A \sigma\left(1-\mathrm{e}^{-2 a / \sigma}\right)\right]=\theta .
$$

The transcendental equation (2.5) can be solved numerically using a root finding algorithm. Since $0<A<1$ and $\sigma>1$, it is straightforward to show that $\mathrm{e}^{-2 a}-$ $A \sigma \mathrm{e}^{-2 a / \sigma}$ is a unimodal function of $a$ and, hence, the maximum number of bump solutions is two. The variation of pulse width with the parameters $\theta$ and $\beta$ is shown in Figure 2.1; the stability of the bumps is analyzed next.

\footnotetext{
${ }^{2}$ These threshold-crossing conditions are necessary but not sufficient for existence of a bump. A rigorous proof of existence, which establishes that activity is superthreshold everywhere within the domain $|x|<a$ and subthreshold for all $|x|>a$, has not been obtained except in special cases [2]. However, it is straightforward to check numerically that these conditions are satisfied.
} 

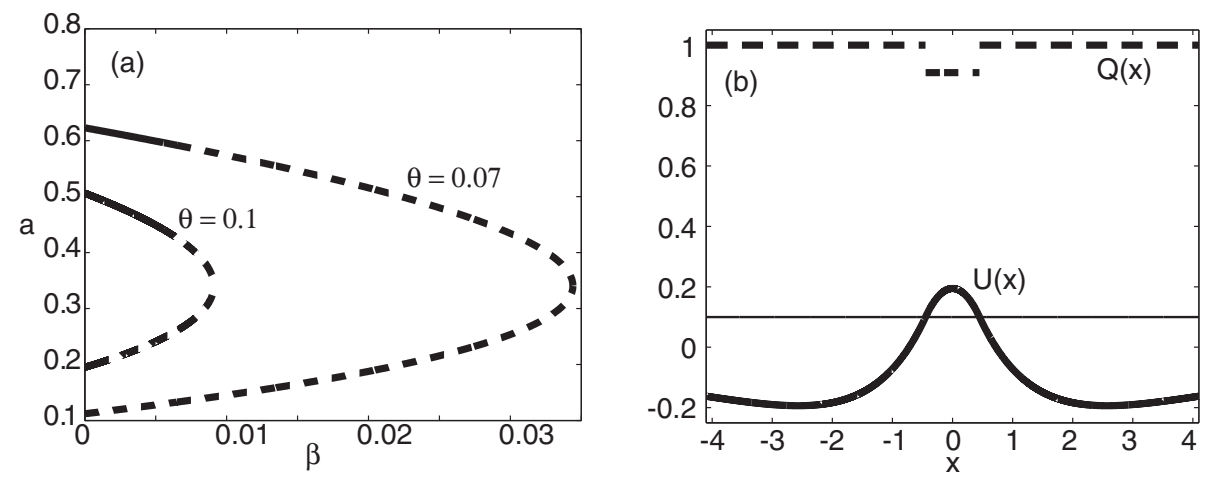

FIG. 2.1. One-dimensional bumps. (a) Plots relating bump width a to amplitude of synaptic depression $\beta$ for different values of $\theta$ using (2.5). The stability analysis of section 3.3 establishes that bumps along the dashed portions of the existence curves are unstable. The solid curves indicate bumps that appear to be numerically stable. Other parameter values are $A=0.6, \sigma=4, \alpha=20$. (b) Bump profile when $\theta=0.1$ and $\beta=0.005$.

2.2. Stability of one-dimensional bumps. In the case of the scalar equation (1.1) it was possible to determine the local stability of a stationary bump by formally differentiating the Heaviside firing rate function inside the convolution integral, which is equivalent to differentiating with respect to the locations of the bump boundary. This is no longer possible for the neural field system (1.6), since the steady-state depression variable $Q(x)$ is a discontinuous function of $x$, reflecting the piecewisesmooth nature of the depression dynamics. One way to handle the discontinuity would be to formally Taylor expand (1.6) about a bump solution in the case of a sigmoid firing rate function and then to take the high-gain limit of the associated Evans function [7]. However, as we have recently shown [21], the size of perturbations for which the linear theory remains valid vanishes in the high-gain limit so that the Evans function approach breaks down; the resulting stability conditions underestimate the effectiveness of synaptic depression in destabilizing a bump. In our previous paper [21], we developed a more careful treatment of local stability that explicitly takes into account the piecewise-smooth nature of the dynamics. Here we present a more detailed version of our 1D analysis in order to lay the groundwork for the stability analysis of $2 \mathrm{D}$ bumps.

Let $u(x, t)=U(x)+\epsilon \psi(x, t)$ and $q(x, t)=Q(x)+\epsilon \varphi(x, t)$. Substituting into the full system (1.6), imposing the stationary bump solutions (2.1) and (2.2), and dividing through by $\epsilon$ gives

$$
\begin{aligned}
\frac{\partial \psi(x, t)}{\partial t}= & -\psi(x, t) \\
& +\frac{1}{\epsilon} \int_{-\infty}^{\infty} w\left(x-x^{\prime}\right) Q\left(x^{\prime}\right)\left[H\left(U\left(x^{\prime}\right)+\epsilon \psi\left(x^{\prime}, t\right)-\theta\right)-H\left(U\left(x^{\prime}\right)-\theta\right)\right] \mathrm{d} x^{\prime} \\
& +\int_{-\infty}^{\infty} w\left(x-x^{\prime}\right) \varphi\left(x^{\prime}, t\right) H\left(U\left(x^{\prime}\right)+\epsilon \psi\left(x^{\prime}, t\right)-\theta\right) \mathrm{d} x^{\prime},
\end{aligned}
$$

$$
\begin{aligned}
\frac{\partial \varphi(x, t)}{\partial t}= & -\frac{\varphi(x, t)}{\alpha}-\frac{\beta}{\epsilon} Q(x)[H(U(x)+\epsilon \psi(x, t)-\theta)-H(U(x)-\theta)] \\
& -\beta \varphi(x, t) H(U(x)+\epsilon \psi(x, t)-\theta) .
\end{aligned}
$$


Denote the perturbations of the bump boundary by $\epsilon \Delta_{ \pm}(t)$ such that

$$
u\left(a+\epsilon \Delta_{+}(t), t\right)=u\left(-a+\epsilon \Delta_{-}(t), t\right)=\theta
$$

for all $t>0$. Taylor expanding these threshold conditions to first order in $\epsilon$, we find that

$$
\Delta_{ \pm}(t) \approx \pm \frac{\psi( \pm a, t)}{\left|U^{\prime}(a)\right|}
$$

We then introduce the auxiliary field

$$
\Phi(x, t)=\int_{-a+\epsilon \Delta_{-}(t)}^{a+\epsilon \Delta_{+}(t)} w\left(x-x^{\prime}\right) \varphi\left(x^{\prime}, t\right) \mathrm{d} x^{\prime} .
$$

The motivation for this is that a small shift in the location of the bump boundary means that in a small neighborhood of the bump boundary the synaptic depression variable will start to switch its steady-state value from $q=1$ to $q=(1+\alpha \beta)^{-1}$ or vice-versa according to (1.6b). That is, $\varphi(x, t)$ will undergo $\mathcal{O}(1 / \epsilon)$ changes over a timescale of $\alpha^{-1}$. However, this doesn't necessarily imply that the bump solution is unstable, since the region over which $\varphi(x, t)=\mathcal{O}(1 / \epsilon)$ may shrink to zero. This is captured by the dynamics of the auxiliary field $\Phi(x, t)$, which will remain $\mathcal{O}(1)$ when $\varphi(x, t)$ is $\mathcal{O}(1 / \epsilon)$ over an infinitesimal interval.

Differentiating (2.9) with respect to time shows that

$$
\begin{aligned}
\frac{\partial \Phi(x, t)}{\partial t}= & \int_{-a+\epsilon \Delta_{-}(t)}^{a+\epsilon \Delta_{+}(t)} w\left(x-x^{\prime}\right) \frac{\partial \varphi\left(x^{\prime}, t\right)}{\partial t} \mathrm{~d} x \\
& +\epsilon w\left(x-a-\epsilon \Delta_{+}(t)\right) \varphi\left(a+\epsilon \Delta_{+}(t), t\right) \dot{\Delta}_{+}(t) \\
& -\epsilon w\left(x+a-\epsilon \Delta_{-}(t)\right) \varphi\left(-a+\epsilon \Delta_{-}(t), t\right) \dot{\Delta}_{-}(t),
\end{aligned}
$$

where $\dot{\Delta}=d \Delta / d t$. Substituting for $\partial \varphi / \partial t$ using (2.7) and replacing the final term on the right-hand side of (2.6) by $\Phi$ leads to the pair of equations

$$
\begin{aligned}
\frac{\partial \psi(x, t)}{\partial t}=- & \psi(x, t)+\Phi(x, t) \\
& +\frac{1}{\epsilon} \int_{-a+\epsilon \Delta_{-}(t)}^{a+\epsilon \Delta_{+}(t)} w\left(x-x^{\prime}\right) Q\left(x^{\prime}\right) \mathrm{d} x^{\prime}-\frac{1}{\epsilon} \int_{-a}^{a} w\left(x-x^{\prime}\right) Q\left(x^{\prime}\right) \mathrm{d} x^{\prime}, \\
\frac{\partial \Phi(x, t)}{\partial t}= & -\left(\alpha^{-1}+\beta\right) \Phi(x, t) \\
& -\frac{\beta}{\epsilon} \int_{-a+\epsilon \Delta_{-}(t)}^{a+\epsilon \Delta_{+}(t)} w\left(x-x^{\prime}\right) Q\left(x^{\prime}\right) H\left(U\left(x^{\prime}\right)+\epsilon \psi\left(x^{\prime}, t\right)-\theta\right) \mathrm{d} x^{\prime} \\
& +\frac{\beta}{\epsilon} \int_{-a+\epsilon \Delta_{-}(t)}^{a+\epsilon \Delta_{+}(t)} w\left(x-x^{\prime}\right) Q\left(x^{\prime}\right) H\left(U\left(x^{\prime}\right)-\theta\right) \mathrm{d} x^{\prime} \\
& +\epsilon w\left(x-a-\epsilon \Delta_{+}(t)\right) \varphi\left(a+\epsilon \Delta_{+}(t), t\right) \dot{\Delta}_{+}(t) \\
& -\epsilon w\left(x+a-\epsilon \Delta_{-}(t)\right) \varphi\left(-a+\epsilon \Delta_{-}(t), t\right) \dot{\Delta}_{-}(t) .
\end{aligned}
$$

We now "linearize" (2.10) and (2.11) by expanding in powers of $\epsilon$ and collecting all $\mathcal{O}(1)$ terms. Note that it is important to keep track of the signs of $\Delta_{ \pm}$when approximating the various integrals, since the stationary solution $Q(x)$ is discontinuous at 
the bump boundary. For example,

$$
\begin{aligned}
\int_{a}^{a+\epsilon \Delta_{+}} w\left(x-x^{\prime}\right) Q\left(x^{\prime}\right) \mathrm{d} x^{\prime} & \approx \epsilon \Delta_{+} \lim _{\epsilon \rightarrow 0^{+}} w\left(x-a-\epsilon \Delta_{+}\right) Q\left(a+\epsilon \Delta_{+}\right) \\
& =\epsilon \Delta_{+} w(x-a) G\left(\Delta_{+}\right)
\end{aligned}
$$

and

$$
\begin{aligned}
\int_{-a+\epsilon \Delta_{-}}^{-a} w\left(x-x^{\prime}\right) Q\left(x^{\prime}\right) \mathrm{d} x^{\prime} & \approx-\epsilon \Delta_{-} \lim _{\epsilon \rightarrow 0^{+}} w\left(x+a-\epsilon \Delta_{-}\right) Q\left(-a+\epsilon \Delta_{-}\right) \\
& =-\epsilon \Delta_{-} w(x+a) G\left(-\Delta_{-}\right)
\end{aligned}
$$

where $G$ is the step function

$$
G(\Delta)=\left\{\begin{array}{cc}
1 & \text { if } \Delta>0 \\
(1+\alpha \beta)^{-1} & \text { if } \Delta<0
\end{array}\right.
$$

Similarly, the two integrals on the right-hand side of (2.11) can be approximated by the expression

$$
\epsilon \Delta_{+}(t) w(x-a) G\left(\Delta_{+}\right) H\left(\Delta_{+}\right)-\epsilon \Delta_{-}(t) w(x+a) G\left(-\Delta_{-}\right) H\left(-\Delta_{-}\right) .
$$

Finally, collecting all $\mathcal{O}(1)$ terms and using (2.8), we obtain the following pseudolinear system of equations:

$$
\begin{aligned}
\frac{\partial \psi(x, t)}{\partial t}= & -\psi(x, t)+\Phi(x, t)+\gamma w(x+a) \psi(-a, t) G(\psi(-a, t)) \\
& +\gamma w(x-a) \psi(a, t) G(\psi(a, t)) \\
\frac{\partial \Phi(x, t)}{\partial t}= & -\left(\alpha^{-1}+\beta\right) \Phi(x, t) \\
& -\beta \gamma w(x+a) \psi(-a, t) G(\psi(-a, t)) H(\psi(-a, t)) \\
& -\beta \gamma w(x-a) \psi(a, t) G(\psi(a, t)) H(\psi(a, t)) .
\end{aligned}
$$

Here

$$
\gamma^{-1}=U^{\prime}(-a)=-U^{\prime}(a)=\frac{w(0)-w(2 a)}{1+\alpha \beta} .
$$

Although it is not possible to determine a complete set of solutions to (2.16), we can simplify the equations under the ansatz that the perturbations $\psi( \pm a, t)$ (or, equivalently, $\left.\Delta_{ \pm}(t)\right)$ do not switch sign for any time $t>0$. In other words, we assume that (2.16) and (2.17) have separable solutions of the form $(\psi(x, t), \Phi(x, t))=$ $\mathrm{e}^{\lambda t}(\psi(x), \Phi(x))$, where $\lambda$ is real and $\psi(x), \Phi(x)$ are bounded continuous functions on $\mathbb{R}$ that decay to zero exponentially as $x \rightarrow \pm \infty$. (The latter reflects the asymptotic properties of the weight distribution $w(x)$.) Under the assumption that $\lambda$ is real, the step functions $H, G$ are time-independent so that there is a common factor $\mathrm{e}^{\lambda t}$ that 
cancels everywhere. We thus obtain the pair of time-independent equations ${ }^{3}$

$$
(\lambda+1) \psi(x)-\Phi(x)=\gamma w(x+a) \psi(-a) G(\psi(-a))+\gamma w(x-a) \psi(a) G(\psi(a)),
$$

$$
\begin{aligned}
\left(\lambda+\alpha^{-1}+\beta\right) \Phi(x)= & -\beta \gamma w(x+a) \psi(-a) G(\psi(-a)) H(\psi(-a)) \\
& -\beta \gamma w(x-a) \psi(a) G(\psi(a)) H(\psi(a)) .
\end{aligned}
$$

One class of solutions to $(2.20)$ is given by $\lambda=-\left(\alpha^{-1}+\beta\right)$ and $\psi(a) \leq 0, \psi(-a) \leq$ 0 . The functions $\psi(x)$ and $\Phi(x)$ are then related according to (2.19). However, such solutions do not contribute to any instabilities. Therefore, we will assume that $\lambda \neq-\left(\alpha^{-1}+\beta\right)$. We can then algebraically eliminate $\Phi(x)$ to obtain the following nonlinear "eigenvalue" equation for $\lambda$ :

$$
\begin{aligned}
(\lambda+1) \psi(x)= & \gamma w(x-a) \psi(a) G(\psi(a))\left[1-\frac{\beta}{\lambda+\alpha^{-1}+\beta} H(\psi(a))\right] \\
& +\gamma w(x+a) \psi(-a) G(\psi(-a))\left[1-\frac{\beta}{\lambda+\alpha^{-1}+\beta} H(\psi(-a))\right] .
\end{aligned}
$$

We now show how solutions to (2.21) can be related to the spectra of a set of compact linear operators, generalizing the analysis of the scalar equation (1.1) by Guo and Chow [16]. Let $\mathcal{C}[-a, a]$ denote the space of bounded, continuous functions on the interval $[-a, a]$. Introduce the set of $\lambda$-dependent linear operators $\mathcal{L}_{m n}(\lambda)$ : $\mathcal{C}[-a, a] \rightarrow \mathcal{C}[-a, a]$ with $m= \pm 1, n= \pm 1$,

$$
\begin{aligned}
\mathcal{L}_{m n}(\lambda) \psi(x)= & \gamma w(x-a) \psi(a)\left[\delta_{m, 1}+\frac{1}{1+\alpha \beta} \delta_{m,-1}\right]\left[1-\frac{\beta}{\lambda+\alpha^{-1}+\beta} \delta_{m, 1}\right] \\
& +\gamma w(x+a) \psi(-a)\left[\delta_{n, 1}+\frac{1}{1+\alpha \beta} \delta_{n,-1}\right]\left[1-\frac{\beta}{\lambda+\alpha^{-1}+\beta} \delta_{n, 1}\right],
\end{aligned}
$$

where $\delta_{m, n}$ is the Kronecker delta. We then consider a corresponding set of eigenvalue problems (for fixed $\lambda$ )

$$
\mu_{n m}(\lambda) \psi(x)=\mathcal{L}_{m n}(\lambda) \psi(x) \quad \text { on } \quad \mathcal{C}[-a, a], \quad m= \pm 1, n= \pm 1 .
$$

Following Guo and Chow [16], it can be shown that $\mathcal{L}_{m n}(\lambda)$ for $\lambda \neq-\left(\alpha^{-1}+\beta\right)$ is a compact linear operator (with respect to the $L^{1}$ norm, say). Hence, the eigenvalues $\mu_{m n}(\lambda)$ of $\mathcal{L}_{m n}(\lambda)$ have only zero as an accumulation point, so that $\mu_{m n}=0$ belongs to the essential spectrum of $\mathcal{L}_{m n}(\lambda)$; the corresponding solutions $\psi(x)$ of $(2.23)$ vanish at the boundaries of the bump, that is, $\psi( \pm a)=0$. The discrete spectrum of $\mathcal{L}_{m n}(\lambda)$ is then obtained by setting $x= \pm a$ in (2.23) and solving the resulting $\lambda$-dependent matrix equation for the pair $\psi( \pm a)$. Having obtained the complete spectrum of each of the linear operators $\mathcal{L}_{m n}(\lambda)$ for fixed $\lambda$, we can then obtain all solutions of (2.21) by solving the corresponding nonlinear equations for $\lambda,{ }^{4}$

$$
\lambda+1=\mu_{n m}(\lambda),
$$

\footnotetext{
${ }^{3}$ It is important to note that our analysis can provide only sufficient conditions for instability but not stability of a bump. This follows from the fact that we are assuming that $\lambda$ is real in order to construct separable solutions of the pseudolinear equations (2.16) and (2.17).

${ }^{4}$ As a slight abuse of notation, we will refer to a solution $\lambda$ of (2.21) as belonging to the discrete (essential) spectrum if the corresponding solution $\mu_{n m}(\lambda)$ of (2.23) belongs to the discrete (essential) spectrum of the linear operator $\mathcal{L}_{m n}(\lambda)$.
} 

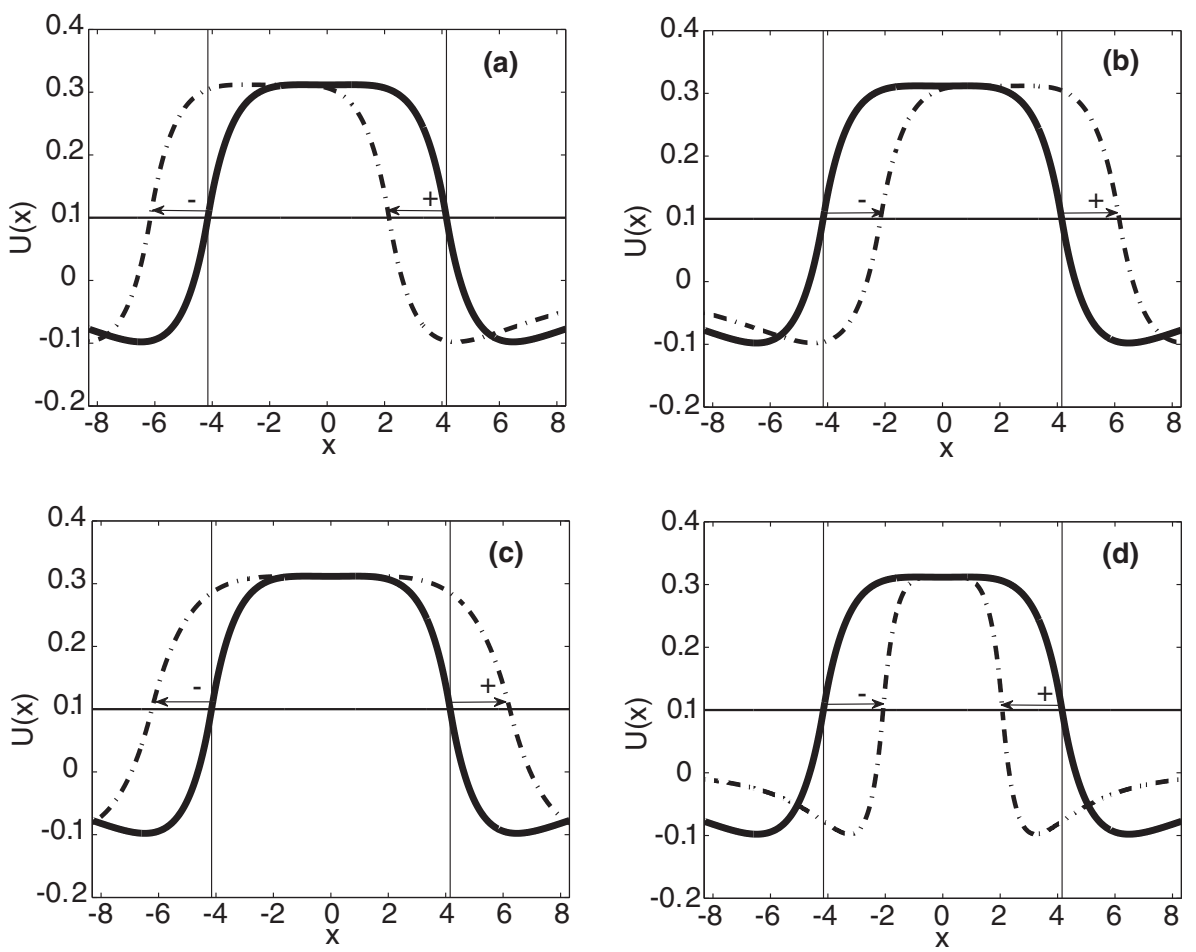

FIG. 2.2. Illustration of different eigenmodes for bump perturbations: (a) left-shift, (b) rightshift, (c) expansion, (d) contraction.

after imposing the appropriate restrictions on the signs of $\psi( \pm a)$ together with the requirement that $\lambda$ is real and $\lambda \neq-\left(\alpha^{-1}+\beta\right)$. In the special case $\mu_{n m}=0$, we see that $\lambda=-1$ and $\psi( \pm a)=0$ irrespective of $n, m$. Thus, the essential spectrum does not contribute to any instabilities. On the other hand, in the case of the discrete spectrum, we require $\operatorname{sign}(\psi(a))=m$ and $\operatorname{sign}(\psi(-a))=n$. Once the eigenvalues have been obtained, the corresponding eigenfunctions are completely determined in terms of $\psi( \pm a)$ and $w(x)$ according to (2.21). The restrictions on the signs of $\psi( \pm a)$ also have a physical interpretation in terms of four different classes of perturbation of the stationary bump solution, which we refer to as expansions $(\psi(-a)>0, \psi(a)>0)$, contractions $(\psi(-a)<0, \psi(a)<0)$, leftward shifts $(\psi(-a)>0, \psi(a)<0)$, and rightward shifts $(\psi(-a)<0, \psi(a)>0)$. (Note, however, that this classification is slightly misleading because if $|\psi(a) / \psi(-a)| \neq 1$, then the width of the bump also changes in the case of shift perturbations.) These basic perturbations are shown in Figure 2.2. We now consider the discrete spectrum in each of the above four cases.

(i) $\psi( \pm a) \geq 0$. Taking $m=n=1$ in (2.23) and rearranging gives

$$
\begin{aligned}
\left(\lambda+\alpha^{-1}+\beta\right)(\lambda+1) \psi(x)= & \gamma w(x+a) \psi(-a)\left(\lambda+\alpha^{-1}\right) \\
& +\gamma w(x-a) \psi(a)\left(\lambda+\alpha^{-1}\right) .
\end{aligned}
$$

Setting $x= \pm a$ and noting that $\psi( \pm a)$ must have the same sign, we have $\psi(a)=$ $\psi(-a)$ with $\lambda$ satisfying the quadratic equation

$$
\left(\lambda+\alpha^{-1}+\beta\right)(\lambda+1)=\left(\lambda+\alpha^{-1}\right)(1+\alpha \beta) \Omega,
$$


where

$$
\Omega=\frac{w(0)+w(2 a)}{w(0)-w(2 a)} .
$$

We have substituted for $\gamma$ using (2.18). It follows that $\lambda=\lambda_{ \pm}$with

$$
\begin{aligned}
\lambda_{ \pm}= & \frac{1}{2}\left[\Omega(1+\alpha \beta)-\left(1+\alpha^{-1}+\beta\right)\right] \\
& \pm \frac{1}{2} \sqrt{\left[\Omega(1+\alpha \beta)-\left(1+\alpha^{-1}+\beta\right)\right]^{2}+4(\Omega-1)\left(\alpha^{-1}+\beta\right)} .
\end{aligned}
$$

The corresponding eigenfunctions represent expansions of the bump and take the form

$$
\left(\begin{array}{c}
\psi(x) \\
\Phi(x)
\end{array}\right)=[w(x+a)+w(x-a)]\left(\begin{array}{c}
1 \\
-\frac{\lambda_{ \pm}+1}{\lambda_{ \pm}+\alpha^{-1}}
\end{array}\right) .
$$

As we illustrate below, for certain ranges of parameters the eigenvalues $\lambda_{ \pm}$form a complex conjugate pair, and thus must be excluded from our analysis since they violate the separability assumption.

(ii) $\psi( \pm a) \leq 0$. Taking $m=-1, n=-1$ in (2.23) gives

$$
(\lambda+1) \psi(x)=\gamma w(x+a) \psi(-a) \frac{1}{1+\alpha \beta}+\gamma w(x-a) \psi(a) \frac{1}{1+\alpha \beta} .
$$

Setting $x= \pm a$ and noting that $\psi( \pm a)$ must have the same sign shows that $\psi(a)=$ $\psi(-a)$ and $\lambda=\lambda_{0}$ with

$$
\lambda_{0}=\Omega-1 .
$$

Hence, there is a single eigenfunction corresponding to a contraction of the bump given by

$$
\left(\begin{array}{c}
\psi(x) \\
\Phi(x)
\end{array}\right)=-\left(\begin{array}{c}
w(x+a)+w(x-a) \\
0
\end{array}\right)
$$

(iii) $\psi(a) \leq 0, \psi(-a) \geq 0$. Taking $m=-1, n=1$ in (2.23) gives

$$
\begin{aligned}
\left(\lambda+\alpha^{-1}+\beta\right)(\lambda+1) \psi(x)= & \gamma w(x+a) \psi(-a)\left(\lambda+\alpha^{-1}\right) \\
& +\gamma w(x-a) \psi(a) \frac{\lambda+\alpha^{-1}+\beta}{1+\alpha \beta} .
\end{aligned}
$$

Setting $x= \pm a$ then yields the matrix equation

$$
\begin{gathered}
\left(\begin{array}{cc}
\Gamma_{\beta}(\lambda)-\gamma w(0)\left(\lambda+\alpha^{-1}\right) & -\gamma\left(\lambda+\alpha^{-1}\right) w(2 a) \\
-\gamma\left(\lambda+\alpha^{-1}\right) w(2 a) & \Gamma_{\beta}(\lambda)-\gamma w(0)\left(\lambda+\alpha^{-1}\right)
\end{array}\right)\left(\begin{array}{c}
\psi(-a) \\
\psi(a)
\end{array}\right) \\
=-\frac{\gamma \alpha \beta \lambda}{1+\alpha \beta}\left(\begin{array}{c}
w(2 a) \psi(a) \\
w(0) \psi(a)
\end{array}\right)
\end{gathered}
$$

where

$$
\Gamma_{\beta}(\lambda)=\left(\lambda+\alpha^{-1}+\beta\right)(\lambda+1) .
$$



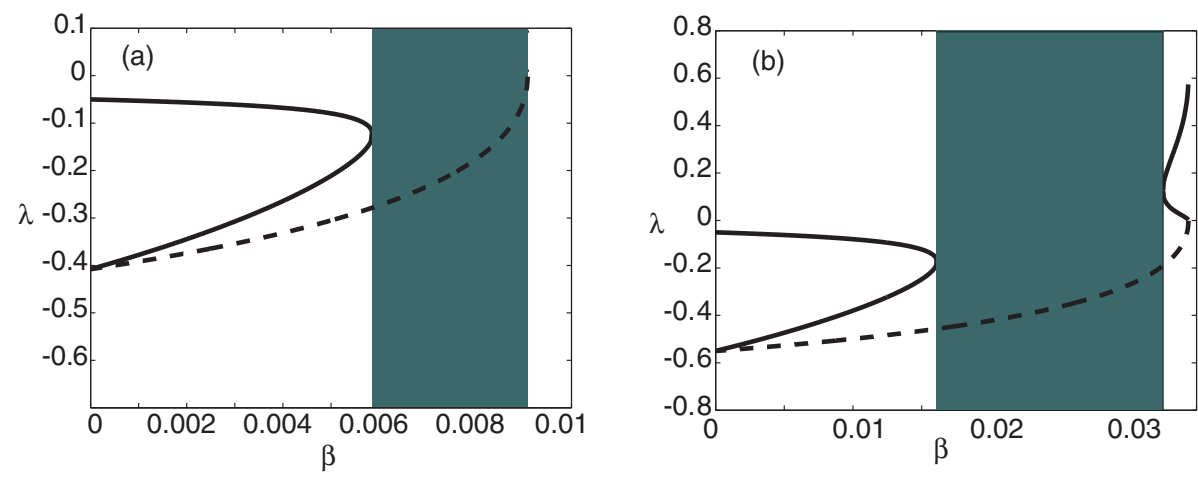

FIG. 2.3. Eigenvalues associated with expansion and contraction perturbations (cases (i) and (ii)). Eigenvalues of the expansion (solid curves) and contraction (dashed curves) perturbations when (a) $\theta=0.1$ and (b) $\theta=0.07$. In the grey regions, the roots of (2.28) are complex, thus violating the ansatz that $\lambda$ is real. Other parameters are $A=0.6, \sigma=4$, and $\alpha=20$.

We thus obtain a quartic characteristic equation for $\lambda$. It is straightforward to show that there always exists a zero eigenvalue $\lambda=0$ with corresponding eigenmode $\psi(-a)=-\psi(a)$. The existence of a zero eigenvalue reflects the translation invariance of the full system (1.6). ${ }^{5}$ In order to calculate the other eigenvalues, we assume that $\beta \ll 1$ (which is consistent with physiological values for the depletion rate of synaptic depression [34,37]) and use perturbation theory. First, setting $\beta=0$ in (2.34) we find that there are three eigenvalues $\lambda_{0}=\Omega-1,0,-\alpha^{-1}$. The first eigenvalue is $\lambda_{0}=\Omega-1$, which can be excluded since the corresponding eigenmode violates the assumption that $\psi( \pm a)$ have opposite sign; the associated solution branch for $\beta>0$ can also be excluded. The second eigenvalue is $\lambda_{0}=0$, which persists when $\beta>0$ (see above). Finally, the third eigenvalue $\lambda_{0}=-\alpha^{-1}$ is doubly degenerate so that one needs to use degenerate perturbation theory in order to determine the splitting of the eigenvalue into two branches as $\beta$ increases from zero; $\lambda$ is a continuous function of $\beta$ on both branches. Again, one of the branches is excluded by requiring that $\psi( \pm a)$ have opposite sign. We conclude that for sufficiently small $\beta$, shift perturbations do not lead to any instabilities.

(iv) $\psi(a) \geq 0, \psi(-a) \leq 0$. As expected from the reflectional symmetry of the original system (1.6) when $w(x)$ is an even function, the spectrum associated with rightward shifts is identical to that of leftward shifts.

We illustrate the above analysis by considering stationary bumps in a network with the Mexican hat weight function (2.4). Specifically, we plot the eigenvalues for each type of perturbation in the case of the wider bump shown in Figure 2.1, which is stable as $\beta \rightarrow 0$. In Figure 2.3, we plot the real eigenvalues associated with expansion and contractions (cases (i) and (ii)) as functions of $\beta$. In the case of contractions, there is a single negative branch that approaches zero at the critical value $\beta=\beta_{c}$. Equations (2.31) and (2.27) imply that the variation in $\lambda$ is due to the $\beta$-dependence of the bump width $a$ such that $\Omega \rightarrow 1$ as $\beta \rightarrow \beta_{c}$. Hence, $a \rightarrow a_{c}$ as $\beta \rightarrow \beta_{c}$, where $a_{c}$ is the width at which the upper and lower existence curves meet in Figure 2.1, that is, $w\left(2 a_{c}\right)=0$ (see also section 2). In the case of

\footnotetext{
${ }^{5}$ Although a small uniform shift of the bump corresponds to $\mathcal{O}(1)$ pointwise changes in the depression variable $q$, these occur over an infinitesimal spatial domain so that the auxiliary variable $\Phi$ is still small. Hence, the response to small uniform shifts is covered by our linear stability analysis.
} 

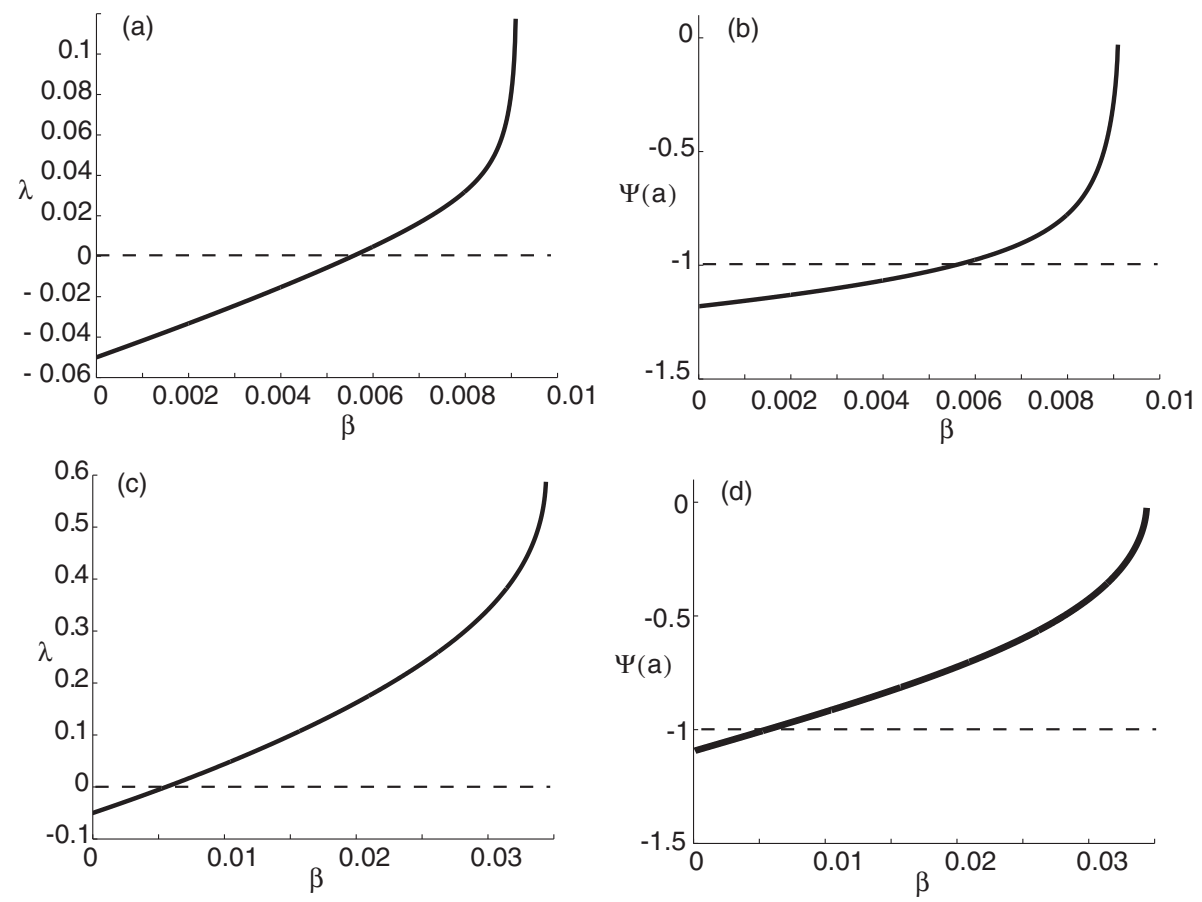

FIG. 2.4. Eigenvalues associated with shift perturbations (cases (iii) and (iv)). (a) Nonzero real eigenvalue plotted as a function of $\beta$ for $\theta=0.1$. Bump is unstable with respect to shifts for sufficiently large $\beta$. (b) Corresponding plot of the ratio $\Psi(a)=\psi(a) / \psi(-a)$ for a leftward shift and $\theta=0.1$. As $\beta$ increases, the ratio approaches zero. (c), (d) Same as (a), (b) for $\theta=0.07$. Other parameters are $A=0.6, \sigma=4, \alpha=20$. Results are the same for a rightward shift on exchanging $x=-a$ and $x=a$.

expansions, there are two negative branches for sufficiently small $\beta$, which annihilate each other at the left-hand side of a forbidden region in which the eigenvalues $\lambda_{ \pm}$ given by (2.28) are complex, so that stability with respect to expansion perturbations cannot be determined. At the other end of this forbidden region there is a jump in the value of the real eigenvalue from which a pair of positive branches $\lambda=\lambda_{ \pm}$emerges. Moreover, the lower expansion branch merges with the contraction branch at the saddle-node bifurcation where the bump comes into existence. That is, $\lambda_{-} \rightarrow 0$ as $\beta \rightarrow \beta_{c}$, which follows from setting $\Omega=1$ in (2.28). In Figure 2.4 we plot the nonzero eigenvalue $\lambda$ for shift perturbations; the other two nonzero solutions to the characteristic equation (2.34) violate the condition that $\psi( \pm a)$ have opposite sign. As $\beta$ increases, the eigenvalue becomes positive, representing destabilization of the bump with respect to shift perturbations. Moreover, Figure 2.4 shows that beyond the point of instability we have $0>\Psi(a) \equiv \psi(a) / \psi(-a)>-1$, so that the width of the bump also increases. Since the shift instability occurs at smaller values of $\beta$ than expansion perturbations, the former dominates bump instabilities in the case of the given Mexican hat weight function.

We summarize our results in Figure 2.5, which shows the parameter space $(\alpha, \beta)$ divided into separate regions, where either no bumps exist (black); bumps are stable with respect to perturbations associated with real eigenvalues (dark grey); or bumps are unstable (light grey). Even when the stability analysis of the expansion mode breaks down, bumps destabilize under shift perturbations. We conclude that strong 


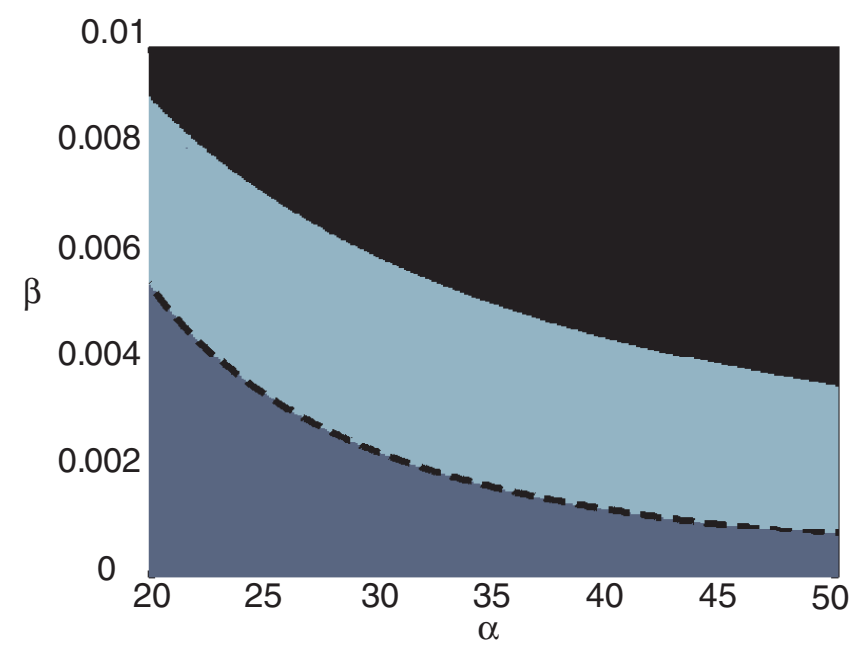

FIG. 2.5. Phase diagram in $(\alpha, \beta)$ parameter space. Black denotes nonexistence of bumps; light grey denotes unstable bumps; dark grey denotes bumps that are stable with respect to perturbations associated with real eigenvalues. The dashed black line denotes location points where numerically stable bumps transition to traveling pulses. Other parameters are $A=0.6, \sigma=4$, and $\theta=0.1$.

enough synaptic depression (large $\beta$ ) can destabilize a stationary bump that would be stable in the absence of depression.

3. Two-dimensional bumps. There have been few studies regarding the existence and stability of standing bumps in 2D neural fields [9, 15, 25, 29]. Laing and Troy [25] were the first to introduce partial differential equation (PDE) methods to study symmetry-breaking of rotationally symmetric bumps. Since then, PDE methods have been used to study the formation of multiple bump solutions, Turing patterns, traveling spots, and labyrinthine patterns in 2D neural fields with local negative feedback $[9,29]$. In addition, standard stability analysis of stimulus-driven 2D neural fields with local linear negative feedback revealed symmetry-breaking breathers [15]. In all of these studies, the eigenmodes associated with instabilities were pure Fourier modes, which were straightforward to pick out using linear stability analysis. In our 2D neural field model, the rotationally nonsymmetric perturbations of bumps possess eigenmodes that are a mixture of pure Fourier modes. This makes the derivation of eigenvalues significantly more complex. Also, we must employ stability analysis techniques that heed the piecewise-smooth nature of the system. Thus, we now extend our analysis of stationary bumps in a $1 \mathrm{D}$ network to derive conditions for the existence and stability of radially symmetric stationary bump solutions of the corresponding 2D piecewise-smooth neural field model

$$
\begin{aligned}
\tau \frac{\partial u(\mathbf{r}, t)}{\partial t} & =-u(\mathbf{r}, t)+\int_{\mathbb{R}^{2}} q\left(\mathbf{r}^{\prime}, t\right) w\left(\left|\mathbf{r}-\mathbf{r}^{\prime}\right|\right) H\left(u\left(\mathbf{r}^{\prime}, t\right)-\theta\right) \mathrm{d} \mathbf{r}^{\prime} \\
\frac{\partial q(\mathbf{r}, t)}{\partial t} & =\frac{1-q(\mathbf{r}, t)}{\alpha}-\beta q(\mathbf{r}, t) H(u(\mathbf{r}, t)-\theta),
\end{aligned}
$$

where $\mathbf{r}=(r, \phi) \in \mathbb{R}^{2}$ and $\left|\mathbf{r}-\mathbf{r}^{\prime}\right|$ denotes Euclidean distance in the plane. 
3.1. Existence. Consider a circularly symmetric bump solution of radius $a$ such that $u(\mathbf{r}, t)=U(r), q(\mathbf{r}, t)=Q(r)$ with $U(a)=\theta$, and

$$
\begin{aligned}
U(r) \gtrless \theta & \text { for } r \lessgtr a, \\
(U(r), Q(r))) \rightarrow(0,1) & \text { as } r \rightarrow \infty .
\end{aligned}
$$

Imposing such constraints on a stationary solution of (3.1) gives

$$
\begin{aligned}
U(r) & =\int_{\mathcal{U}} Q\left(r^{\prime}\right) w\left(\left|\mathbf{r}-\mathbf{r}^{\prime}\right|\right) \mathrm{d} \mathbf{r}^{\prime}, \\
Q(r) & =(1+\alpha \beta H(U(r)-\theta))^{-1},
\end{aligned}
$$

where $\mathcal{U}=\{\mathbf{r}=(r, \phi): r \leq a\}$ is the domain on which the bump is superthreshold. Substituting (3.3) back into (3.2) yields

$$
(1+\alpha \beta) U(r)=\Pi(a, r),
$$

where

$$
\Pi(a, r)=\int_{0}^{2 \pi} \int_{0}^{a} w\left(\left|\mathbf{r}-\mathbf{r}^{\prime}\right|\right) r^{\prime} \mathrm{d} r^{\prime} \mathrm{d} \phi^{\prime} .
$$

We can calculate the double integral in (3.5) using the Hankel transform and Bessel function identities, as in $[14,29]$. Thus, we find that

$$
\Pi(a, r)=2 \pi a \int_{0}^{\infty} \hat{w}(\rho) J_{0}(r \rho) J_{1}(a \rho) \mathrm{d} \rho,
$$

where $\hat{w}(\rho)$ is the Hankel transform of $w$.

For the sake of illustration consider a Mexican hat weight distribution given by a combination of modified Bessel functions of the second kind [14, 26, 29]:

$$
w(r)=\frac{2}{3 \pi}\left(K_{0}(r)-K_{0}(2 r)-A\left(K_{0}(r / \sigma)-K_{0}(2 r / \sigma)\right)\right) .
$$

Such a weight function is qualitatively similar to a difference of exponential weight functions $w(r)=(2 \pi)^{-1}\left(\mathrm{e}^{-r}-A \mathrm{e}^{-r / \sigma}\right)$. Moreover, following previous studies of $2 \mathrm{D}$ neural field models [20, 25, 26, 29, 35], we can transform system (3.1) into a fourthorder PDE, which is computationally less expensive to evaluate. Using the fact that the corresponding Hankel transform of $K_{0}(s r)$ is $\mathcal{H}(\rho, s)=\left(\rho^{2}+s^{2}\right)^{-1}$, we have

$$
\widehat{w}(\rho)=\frac{2}{3 \pi}(\mathcal{H}(\rho, 1)-\mathcal{H}(\rho, 2)-A(\mathcal{H}(\rho, 1 / \sigma)-\mathcal{H}(\rho, 2 / \sigma))) .
$$

Thus, the integral (3.6) can be evaluated explicitly by substituting (3.8) into (3.6) and using the identity

$$
\int_{0}^{\infty} \frac{1}{\rho^{2}+s^{2}} J_{0}(r \rho) J_{1}(a \rho) \mathrm{d} \rho \equiv \mathcal{I}(a, r, s)=\left\{\begin{array}{cc}
\frac{1}{s} I_{1}(s a) K_{0}(s r), & r>a, \\
\frac{1}{a s^{2}}-\frac{1}{s} I_{0}(s r) K_{1}(s a), & r<a,
\end{array}\right.
$$

where $I_{\nu}$ is the modified Bessel function of the first kind of order $\nu$. Thus, the stationary bump $U(r)$ given by (3.4) has the form

$$
U(r)=\frac{4 a}{3(1+\alpha \beta)}(\mathcal{I}(a, r, 1)-\mathcal{I}(a, r, 2)-A(\mathcal{I}(a, r, 1 / \sigma)-\mathcal{I}(a, r, 2 / \sigma)))
$$



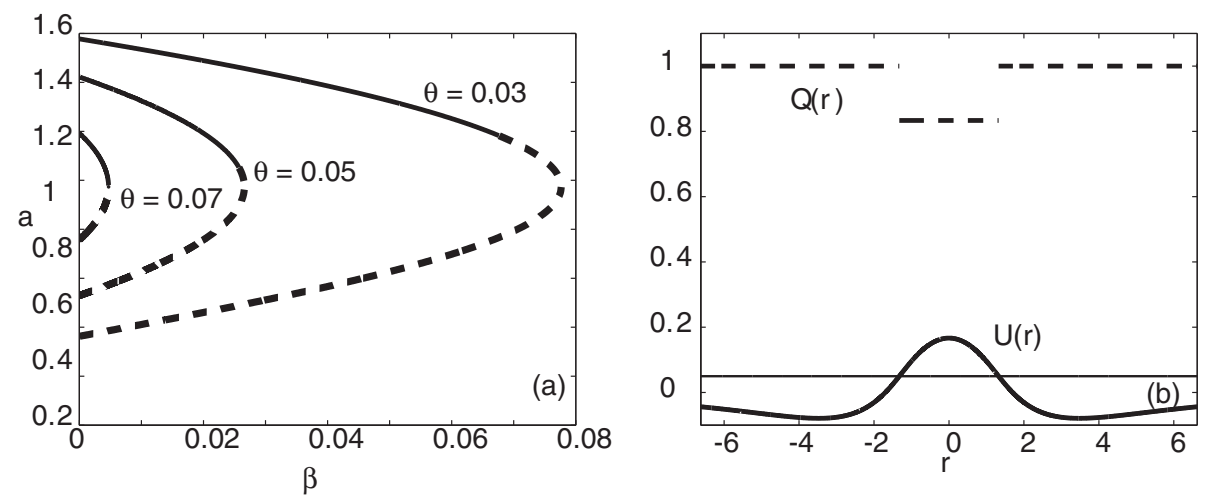

FIG. 3.1. Two-dimensional bumps. (a) Plots relating bump radius a to amplitude of synaptic depression $\beta$ for various values of threshold $\theta$ based on (3.10). Dashed curves indicate bumps that are unstable with respect to $D_{1}$ symmetric perturbations, whereas solid curves indicate bumps that appear to be numerically stable. Other parameters are $A=0.3, \sigma=4, \alpha=20$. (b) Bump profile when $\theta=0.05, \alpha=20, \beta=0.01$.

The bump radius may then be computed by finding the roots $a$ of the equation

$$
(1+\alpha \beta) \theta=\Pi(a)
$$

with

$$
\begin{aligned}
\Pi(a) \equiv \Pi(a, a)=\frac{4 a}{3}( & I_{1}(a) K_{0}(a)-\frac{1}{2} I_{1}(2 a) K_{0}(2 a) \\
& \left.-A\left(\sigma I_{1}(a / \sigma) K_{0}(a / \sigma)-\frac{\sigma}{2} I_{1}(2 a / \sigma) K_{0}(2 a / \sigma)\right)\right) .
\end{aligned}
$$

We typically find a maximum of two bump solutions as illustrated in Figure 3.1, where the bump radius $a$ is plotted as a function of depression strength $\beta$ for various thresholds $\theta$.

3.2. Stability. We now analyze the linear stability of radially symmetric $2 \mathrm{D}$ bump solutions. As in the case of $1 \mathrm{D}$ bumps, we must consider the sign of the perturbations of the bump boundary when carrying out the pseudolinearization. However, there are now an infinite number of cases to consider with regard to how perturbations subdivide the continuum boundary of a $2 \mathrm{D}$ bump.

Let $u(\mathbf{r}, t)=U(r)+\epsilon \psi(\mathbf{r}, t)$ and $q(\mathbf{r}, t)=Q(r)+\epsilon \varphi(\mathbf{r}, t)$. Substituting into the full 2D system (3.1) and imposing the stationary bump solutions (3.2) and (3.3) gives

$$
\begin{aligned}
\frac{\partial \psi(\mathbf{r}, t)}{\partial t}= & -\psi(\mathbf{r}, t) \\
& +\frac{1}{\epsilon} \int_{\mathbb{R}^{2}} w\left(\left|\mathbf{r}-\mathbf{r}^{\prime}\right|\right) Q\left(r^{\prime}\right)\left[H\left(U\left(r^{\prime}\right)+\epsilon \psi\left(\mathbf{r}^{\prime}, t\right)-\theta\right)-H\left(U\left(r^{\prime}\right)-\theta\right)\right] \mathrm{d} \mathbf{r}^{\prime} \\
& +\int_{\mathbb{R}^{2}} w\left(\left|\mathbf{r}-\mathbf{r}^{\prime}\right|\right) \varphi\left(\mathbf{r}^{\prime}, t\right) H\left(U\left(r^{\prime}\right)+\epsilon \psi\left(\mathbf{r}^{\prime}, t\right)-\theta\right) \mathrm{d} \mathbf{r}^{\prime} \\
\frac{\partial \varphi(\mathbf{r}, t)}{\partial t}= & -\frac{\varphi(\mathbf{r}, t)}{\alpha}-\frac{\beta}{\epsilon} Q(r)[H(U(r)+\epsilon \psi(\mathbf{r}, t)-\theta)-H(U(r)-\theta)] \\
- & \beta \varphi(\mathbf{r}, t) H(U(r)+\epsilon \psi(\mathbf{r}, t)-\theta)
\end{aligned}
$$


If $\epsilon \Delta(\phi, t)$ denotes the perturbation of the circular bump boundary at polar coordinate $(a, \phi)$, then

$$
u(a+\epsilon \Delta(\phi, t), \phi, t)=\theta
$$

for $\phi \in[0,2 \pi)$ and all $t>0$. Following our analysis of the 1D case (section 3), we introduce the auxiliary field

$$
\Phi(\mathbf{r}, t)=\int_{0}^{2 \pi} \int_{0}^{a+\epsilon \Delta\left(\phi^{\prime}, t\right)} w\left(\left|\mathbf{r}-\mathbf{r}^{\prime}\right|\right) \varphi\left(\mathbf{r}^{\prime}, t\right) \mathrm{d} r^{\prime} \mathrm{d} \phi^{\prime} .
$$

Differentiating $\Phi(\mathbf{r}, t)$ with respect to $t$ and combining this with (3.12) and (3.13) leads to the pair of equations

$$
\begin{aligned}
\frac{\partial \psi(\mathbf{r}, t)}{\partial t}= & -\psi(\mathbf{r}, t)+\Phi(\mathbf{r}, t)+\frac{1}{\epsilon} \int_{0}^{2 \pi} \int_{0}^{a+\epsilon \Delta\left(\phi^{\prime}, t\right)} w\left(\left|\mathbf{r}-\mathbf{r}^{\prime}\right|\right) Q\left(r^{\prime}\right) r^{\prime} \mathrm{d} r^{\prime} \mathrm{d} \phi^{\prime} \\
& -\frac{1}{\epsilon} \int_{0}^{2 \pi} \int_{0}^{a} w\left(\left|\mathbf{r}-\mathbf{r}^{\prime}\right|\right) Q\left(r^{\prime}\right) r^{\prime} \mathrm{d} r^{\prime} \mathrm{d} \phi^{\prime}, \\
\frac{\partial \Phi(\mathbf{r}, t)}{\partial t}= & -\left(\alpha^{-1}+\beta\right) \Phi(\mathbf{r}, t)-\frac{\beta}{\epsilon} \int_{0}^{2 \pi} \int_{0}^{a+\epsilon \Delta\left(\phi^{\prime}, t\right)} w\left(\left|\mathbf{r}-\mathbf{r}^{\prime}\right|\right) Q\left(r^{\prime}\right) \\
& \times\left[H\left(U\left(\mathbf{r}^{\prime}, t\right)+\epsilon \psi\left(\mathbf{r}^{\prime}, t\right)-\theta\right)-H\left(U\left(\mathbf{r}^{\prime}, t\right)-\theta\right)\right] r^{\prime} \mathrm{d} r^{\prime} \mathrm{d} \phi^{\prime} \\
& +\epsilon \int_{0}^{2 \pi} w\left(\left|\mathbf{r}-\mathbf{r}^{\prime}\right|\right) \varphi\left(\mathbf{r}^{\prime}, t\right) \dot{\Delta}\left(\phi^{\prime}, t\right) \mathrm{d} r^{\prime} \mathrm{d} \phi^{\prime} .
\end{aligned}
$$

We now expand these equations in powers of $\epsilon$ and collect all $\mathcal{O}(1)$ terms. It is important to keep track of the sign of $\Delta(\phi, t)$ at all values of $\phi$ when approximating the integrals, since $Q(r)$ is discontinuous on the boundary. For example,

$$
\begin{aligned}
\int_{0}^{2 \pi} \int_{a}^{a+\epsilon \Delta\left(\phi^{\prime}, t\right)} w\left(\left|\mathbf{r}-\mathbf{r}^{\prime}\right|\right) Q\left(r^{\prime}\right) r^{\prime} \mathrm{d} r^{\prime} \mathrm{d} \phi^{\prime} & \approx \epsilon a \int_{\mathcal{A}_{+}(t)} \Delta\left(\phi^{\prime}, t\right) w(|\mathbf{r}-|) \mathrm{d} \phi^{\prime} \\
& +\epsilon \frac{a}{1+\alpha \beta} \int_{\mathcal{A}_{-}(t)} \Delta\left(\phi^{\prime}, t\right) w(|\mathbf{r}-|) \mathrm{d} \phi^{\prime}
\end{aligned}
$$

where $=\left(a, \phi^{\prime}\right)$ and the domain $\mathcal{A}_{+}(t)\left(\mathcal{A}_{-}(t)\right)$ defines the intervals in $\phi$ over which the perturbation $\Delta(\phi, t)>0(\Delta(\phi, t)<0)$ at time $t>0$. We have used the fact that in the region $\mathcal{A}_{+}(t)\left(\mathcal{A}_{-}(t)\right)$, we approach the stationary bump boundary from the exterior (interior) of the bump in the limit $\epsilon \rightarrow 0^{+}$so that $Q=1(Q=1 /(1+\alpha \beta))$. Likewise, the first integral on the right-hand side of (3.16) can be approximated by $\epsilon a \int_{\mathcal{A}_{+}} \Delta\left(\phi^{\prime}, t\right) w(|\mathbf{r}-|) \mathrm{d} \phi^{\prime}$. Finally, we use the approximation

$$
\begin{aligned}
\theta & =u(a+\epsilon \Delta(\phi, t), \phi, t)=U(a+\epsilon \Delta(\phi, t))+\epsilon \psi(a+\epsilon \Delta(\phi, t), \phi, t), \\
& \approx U(a)+\epsilon U^{\prime}(a) \Delta(\phi, t)+\epsilon \psi(a, \phi),
\end{aligned}
$$

and $U(a)=\theta$ so that

$$
\Delta(\phi, t) \approx \frac{\psi(a, \phi, t)}{\left|U^{\prime}(a)\right|}
$$


to lowest order in $\epsilon$. This leads to the following pair of equations:

$$
\begin{aligned}
\frac{\partial \psi(\mathbf{r}, t)}{\partial t}= & -\psi(\mathbf{r}, t)+\Phi(\mathbf{r}, t)+a \gamma \int_{\mathcal{A}_{+}(t)} \psi\left(a, \phi^{\prime}, t\right) w(|\mathbf{r}-|) \mathrm{d} \phi^{\prime} \\
& +\frac{a \gamma}{1+\alpha \beta} \int_{\mathcal{A}_{-}(t)} \psi\left(a, \phi^{\prime}, t\right) w(|\mathbf{r}-|) \mathrm{d} \phi^{\prime}, \\
\frac{\partial \Phi(\mathbf{r}, t)}{\partial t}= & -\left(\alpha^{-1}+\beta\right) \Phi(\mathbf{r}, t)-a \gamma \beta \int_{\mathcal{A}_{+}(t)} \psi\left(a, \phi^{\prime}, t\right) w(|\mathbf{r}-|) \mathrm{d} \phi^{\prime} .
\end{aligned}
$$

Here

$$
\gamma^{-1}=\left|U^{\prime}(a)\right|=\frac{2 \pi a}{1+\alpha \beta} \int_{0}^{\infty} \rho \widehat{w}(\rho) J_{1}(a \rho) J_{1}(a \rho) \mathrm{d} \rho,
$$

which, for the Mexican hat weight function (3.7), can be explicitly computed as

$$
\begin{aligned}
\left|U^{\prime}(a)\right|=\frac{4 a}{3(1+\alpha \beta)} & \left(I_{1}(a) K_{1}(a)-I_{1}(2 a) K_{1}(2 a)\right. \\
& \left.-A\left(I_{1}(a / \sigma) K_{1}(a / \sigma)-I_{1}(2 a / \sigma) K_{1}(2 a / \sigma)\right)\right) .
\end{aligned}
$$

Equations (3.18) and (3.19) imply that the local stability of a stationary bump solution depends on solutions to a system of pseudolinear equations. As in the 1D case, we can obtain a class of solutions under the ansatz that the perturbation $\psi(a, \phi, t)$ (equivalently, $\Delta(\phi, t))$ does not switch sign at each $\phi$ for any time $t$. Thus, we assume (3.18) and (3.19) have separable solutions $(\psi(\mathbf{r}, t), \Phi(\mathbf{r}, t))=\mathrm{e}^{\lambda t}(\psi(\mathbf{r}), \Phi(\mathbf{r}))$, where $\lambda$ is real and $(\psi(\mathbf{r}), \Phi(\mathbf{r}))$ are bounded continuous functions that decay to zero exponentially as $|\mathbf{r}| \rightarrow \infty$. Under this assumption, the domains $\mathcal{A}_{ \pm}$are constant in time, so there is a common factor $\mathrm{e}^{\lambda t}$ that cancels everywhere. In a similar fashion to the analysis of $1 \mathrm{D}$ bumps, one class of solution is given by $\lambda=-\left(\alpha^{-1}+\beta\right)$ and $\psi(a, \phi) \leq 0$ for all $\phi$. However, this does not contribute to any instabilities. Therefore, suppose that $\lambda \neq-\left(\alpha^{-1}+\beta\right)$. We can then eliminate $\Phi(x)$ to obtain a nonlinear "eigenvalue" equation for $\lambda$ of the form

$$
\begin{aligned}
(\lambda+1) \psi(\mathbf{r})= & \frac{a \gamma\left(\lambda+\alpha^{-1}\right)}{\lambda+\alpha^{-1}+\beta} \int_{\mathcal{A}_{+}} \psi\left(a, \phi^{\prime}\right) w(|\mathbf{r}-|) \mathrm{d} \phi^{\prime} \\
& +\frac{a \gamma}{1+\alpha \beta} \int_{\mathcal{A}_{-}} \psi\left(a, \phi^{\prime}\right) w(|\mathbf{r}-|) \mathrm{d} \phi^{\prime} .
\end{aligned}
$$

We could now proceed along lines similar to the 1D case by reformulating the problem in terms of finding the spectra of a set of compact linear operators acting on continuous, bounded functions $\psi(r, \phi)$ defined on the disc of radius $r \leq a$ with the sign of $\psi(a, \phi), \phi \in[0,2 \pi)$, prescribed. However, here we simply summarize the results. First, one class of solutions to (3.22) consists of functions $\psi(\mathbf{r})$ that vanish on the boundary, $\psi(a, \phi)=0$ for all $\phi$ such that $\lambda=-1$. (It also follows that $\Phi(x) \equiv 0$.) This belongs to the essential spectrum, which does not contribute to any instabilities. The discrete spectrum for given $\mathcal{A}_{ \pm}$is then determined by setting $r=a$ in equation (3.22):

$$
\begin{aligned}
(\lambda+1) \psi(a, \phi)= & \frac{a \gamma\left(\lambda+\alpha^{-1}\right)}{\lambda+\alpha^{-1}+\beta} \int_{\mathcal{A}_{+}} \psi\left(a, \phi^{\prime}\right) w\left(2 a \sin \frac{\phi-\phi^{\prime}}{2}\right) \mathrm{d} \phi^{\prime} \\
& +\frac{a \gamma}{1+\alpha \beta} \int_{\mathcal{A}_{-}} \psi\left(a, \phi^{\prime}\right) w\left(2 a \sin \frac{\phi-\phi^{\prime}}{2}\right) \mathrm{d} \phi^{\prime}
\end{aligned}
$$


where we have simplified the argument of $w(r)$ using

$$
\left|(a, \phi)-\left(a, \phi^{\prime}\right)\right|=\sqrt{\left(a \sin \phi-a \sin \phi^{\prime}\right)^{2}+\left(a \cos \phi-a \cos \phi^{\prime}\right)^{2}}=2 a \sin \frac{\phi-\phi^{\prime}}{2} .
$$

There are three classes of solution to (3.23): (i) radially symmetric expansions such that $\psi(a, \phi)=\psi(a)>0$ for $\phi \in[0,2 \pi)$; (ii) radially symmetric contractions such that $\psi(a, \phi)=\psi(a)<0$ for $\phi \in[0,2 \pi)$; and (iii) radially nonsymmetric perturbations for which $\psi(a, \phi)$ changes sign as a function of $\phi$.

In the limit $\beta \rightarrow 0,(3.23)$ reduces to the simpler form

$$
(\lambda+1) \psi(a, \phi)=a \gamma \int_{0}^{2 \pi} \psi\left(a, \phi^{\prime}\right) w\left(2 a \sin \frac{\phi-\phi^{\prime}}{2}\right) \mathrm{d} \phi^{\prime} .
$$

The eigenmodes are then given by pure Fourier modes $\psi(a, \phi)=\Delta_{n}(\phi) \equiv c_{n} \mathrm{e}^{i n \phi}+$ $\bar{c}_{n} \mathrm{e}^{-i n \phi}$, where $\bar{c}_{n}$ denotes the complex conjugate of $c_{n}$ for integer $n$, with corresponding real eigenvalues $\lambda_{n}=-1+\gamma \mu_{n}$,

$$
\mu_{n}=2 a \int_{0}^{2 \pi} w(2 a \sin \phi) \mathrm{e}^{-2 i n \phi} d \phi .
$$

Some examples of low-order Fourier eigenmodes $\Delta_{n}(\phi)$ are shown in Figure 3.2, together with the associated boundary domains $\mathcal{A}_{ \pm}$. It can be seen that the $n$th order boundary perturbation has $D_{n}$ symmetry, meaning the resulting solution has the $n$ reflectional and rotational symmetries of the dihedral group $D_{n}$. As $\beta$ is increased from zero, only the zeroth-order eigenmodes persist (expansions and contractions), whereas the nonradially symmetric eigenmodes become a mixture of Fourier modes:

$$
\psi(a, \phi)=\sum_{n=-\infty}^{\infty} c_{n}(a) \mathrm{e}^{i n \phi} .
$$

We find numerically, at least for sufficiently small $\beta$, that the eigenmodes still have $D_{n}$ symmetry for $n \geq 1$, but the $\mathcal{A}_{+}$subdomains are now larger than the $\mathcal{A}_{-}$subdomains (see below).

(i) $\psi(a, \phi)=\psi(a)>0$. In this case, (3.23) becomes

$$
(\lambda+1) \psi(a)=\frac{a \gamma\left(\lambda+\alpha^{-1}\right) \psi(a)}{\lambda+\alpha^{-1}+\beta} \int_{0}^{2 \pi} w\left(2 a \sin \frac{\phi-\phi^{\prime}}{2}\right) \mathrm{d} \phi^{\prime},
$$

where we have used the fact that $\mathcal{A}_{+}=[0,2 \pi), \mathcal{A}_{-}$is empty, and $\psi(a, \phi)$ is constant in $\phi$. Therefore, the right-hand side of (3.26) is independent of $\phi$, and $\lambda$ satisfies the quadratic equation

$$
\left(\lambda+\alpha^{-1}+\beta\right)(\lambda+1)=\left(\lambda+\alpha^{-1}\right)(1+\alpha \beta) \Omega_{0},
$$

where

$$
\Omega_{0}=\frac{\mu_{0}(a)}{(1+\alpha \beta)\left|U^{\prime}(a)\right|}, \quad \mu_{0}(a)=2 a \int_{0}^{\pi} w(2 a \sin \phi) \mathrm{d} \phi .
$$

For the Mexican hat weight function (3.7), we can use

$$
\int_{0}^{\pi} K_{0}(2 a \sin \phi) \mathrm{d} \phi=\pi I_{0}(a) K_{0}(a)
$$



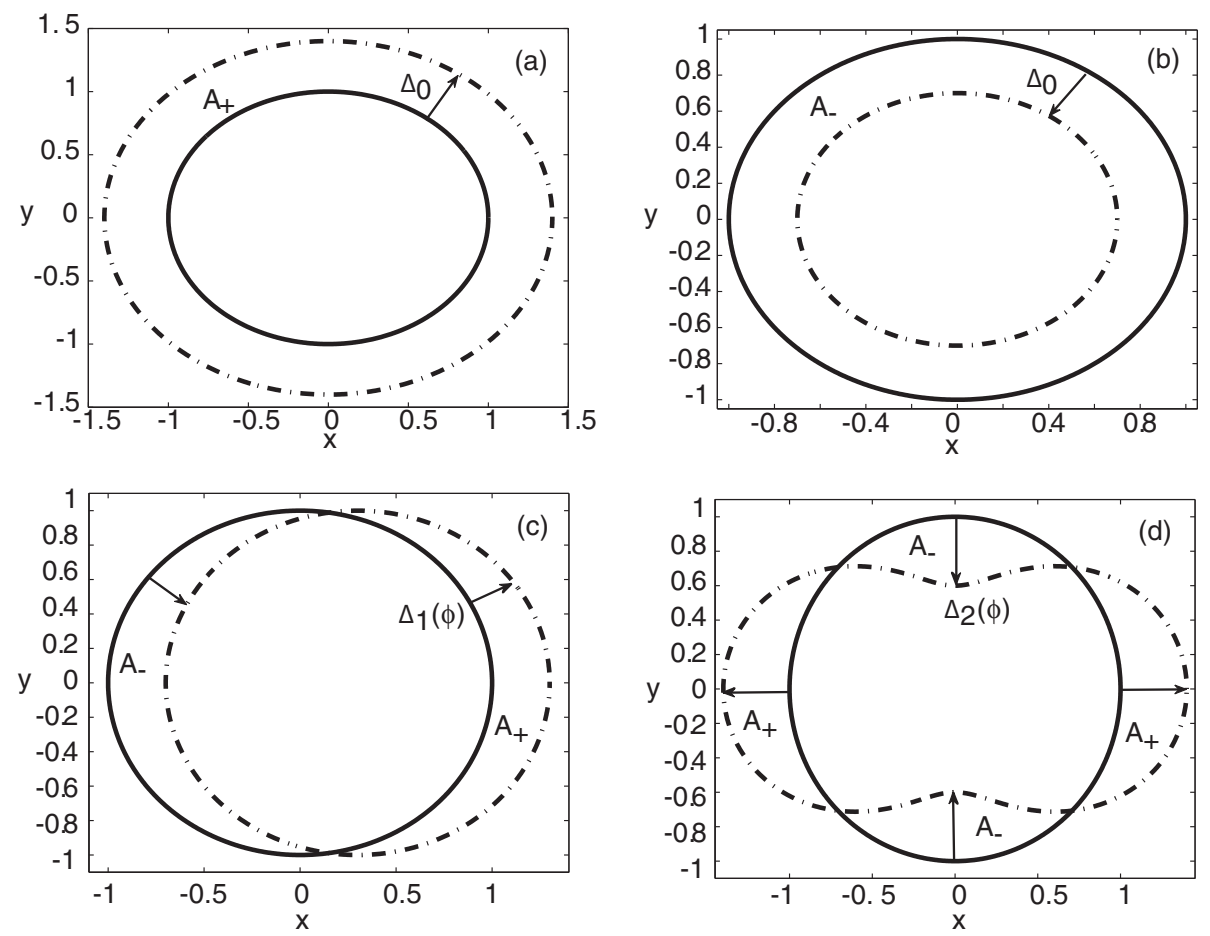

FIG. 3.2. Low-order perturbations of a radially symmetric $2 D$ bump. (a) Expansion $\left(\Delta_{0}>0\right)$; (b) contraction $\left(\Delta_{0}<0\right)$; (c) $D_{1}$-symmetric shift $\Delta_{1}(\phi)$; (d) $D_{2}$-symmetric perturbation $\Delta_{2}(\phi)$.

to calculate

$$
\begin{aligned}
\mu_{0}(a)=\frac{4 a}{3}( & I_{0}(a) K_{0}(a)-I_{0}(2 a) K_{0}(2 a) \\
& \left.\quad-A\left(I_{0}(a / \sigma) K_{0}(a / \sigma)-I_{0}(2 a / \sigma) K_{0}(2 a / \sigma)\right)\right) .
\end{aligned}
$$

It follows that $\lambda=\lambda_{0}^{ \pm}$with

$$
\begin{aligned}
\lambda_{0}^{ \pm}= & \frac{1}{2}\left[\Omega_{0}(1+\alpha \beta)-\left(1+\alpha^{-1}+\beta\right)\right] \\
& \pm \frac{1}{2} \sqrt{\left[\Omega_{0}(1+\alpha \beta)-\left(1+\alpha^{-1}+\beta\right)\right]^{2}+4\left(\Omega_{0}-1\right)\left(\alpha^{-1}+\beta\right)} .
\end{aligned}
$$

The associated eigenmodes correspond to an expansion of the bump.

(ii) $\psi(a, \phi)=\psi(a)<0$. In this case, (3.23) becomes

$$
(\lambda+1) \psi(a)=\frac{a \gamma \psi(a)}{1+\alpha \beta} \int_{0}^{2 \pi} w\left(2 a \sin \frac{\phi-\phi^{\prime}}{2}\right) \mathrm{d} \phi^{\prime},
$$

where we have used the fact that $\mathcal{A}_{+}$is empty, $\mathcal{A}_{-}=[0,2 \pi)$, and $\psi(a, \phi)$ is constant in $\phi$. Therefore, the right-hand side of (3.31) is independent of $\phi$ and $\lambda=\lambda_{0}$ with

$$
\lambda_{0}=\Omega_{0}-1
$$

The associated eigenmode corresponds to a contraction of the bump. 
(iii) $\psi(a, \phi)$ radially nonsymmetric. In this final case, the characteristic equation (3.23) involves integrals over subdomains of $[0,2 \pi)$ and is no longer a standard Fredholm integral equation. Hence, as we have already indicated, eigenmodes will be more complicated than the pure Fourier modes $\mathrm{e}^{i n \phi}$ found in previous studies of bump instabilities in two dimensions [14, 15, 25, 29]. This is due to the faster growth of the lobes of the perturbation $\psi(a, \phi)$ that are superthreshold versus those that are subthreshold. There is then no systematic way of finding the exact general solutions of the spectral problem (3.23), and we must resort to numerical solutions (see below). However, since the underlying system (3.1) is translationally symmetric, the $2 \mathrm{D}$ bump solution given by (3.2) and (3.3) should be invariant with respect to uniform translations. We can check this fact by explicitly showing that the pure shift eigenmode is neutrally stable, as it usually is in studies of the stability of $1 \mathrm{D}$ and $2 \mathrm{D}$ bumps $[2,24,29]$. Specifically, we wish to verify that there is a solution to (3.23) that moves the bump, while keeping its profile invariant such that $\lambda=0$. Thus, upon setting $\lambda=0$ in (3.23), we find that

$$
\psi(a, \phi)=\frac{a \gamma}{1+\alpha \beta} \int_{0}^{2 \pi} \psi\left(a, \phi^{\prime}\right) w\left(2 a \sin \frac{\phi-\phi^{\prime}}{2}\right) \mathrm{d} \phi^{\prime} .
$$

If we now assume that $\psi(a, \phi)=\mathrm{e}^{i \phi}$, a pure shift eigenfunction, and make the change of variables $\phi^{\prime}=\phi-2 \phi^{\prime}$, then (3.33) becomes

$$
\frac{1+\alpha \beta}{2 a \gamma}=\int_{0}^{\pi} \mathrm{e}^{-2 i \phi^{\prime}} w\left(2 a \sin \phi^{\prime}\right) \mathrm{d} \phi^{\prime}
$$

(after rescaling $\phi)$. Notice that the right-hand side of (3.34) is real since

$$
\operatorname{Im}\left\{\int_{0}^{\pi} \mathrm{e}^{-2 i \phi^{\prime}} w\left(2 a \sin \phi^{\prime}\right) \mathrm{d} \phi^{\prime}\right\}=\int_{0}^{\pi} \sin \left(2 \phi^{\prime}\right) w\left(2 a \sin \phi^{\prime}\right) \mathrm{d} \phi^{\prime}=0,
$$

owing to the integrand being odd-symmetric about $\pi / 2$. Therefore,

$$
\frac{1+\alpha \beta}{2 a \gamma}=\int_{0}^{\pi} \cos (2 \phi) w(2 a \sin \phi) \mathrm{d} \phi .
$$

For the Mexican hat weight function (3.7), we can use the identity

$$
\int_{0}^{\pi} K_{0}(2 a \sin \phi) \cos (2 \phi) \mathrm{d} \phi=\pi I_{1}(a) K_{1}(a)
$$

to compute

$$
\begin{aligned}
\int_{0}^{\pi} \cos (2 \phi) w(2 a \sin \phi) \mathrm{d} \phi=\frac{2}{3} & \left(I_{1}(a) K_{1}(a)-I_{1}(2 a) K_{1}(2 a)\right. \\
& \left.\quad-A\left(I_{1}(a / \sigma) K_{1}(a / \sigma)-I_{1}(2 a / \sigma) K_{1}(2 a / \sigma)\right)\right),
\end{aligned}
$$

which we plug into (3.35) so that it becomes

$$
\begin{aligned}
\gamma^{-1}=\frac{4 a}{3(1+\alpha \beta)} & \left(I_{1}(a) K_{1}(a)-I_{1}(2 a) K_{1}(2 a)\right. \\
& \left.-A\left(I_{1}(a / \sigma) K_{1}(a / \sigma)-I_{1}(2 a / \sigma) K_{1}(2 a / \sigma)\right)\right),
\end{aligned}
$$



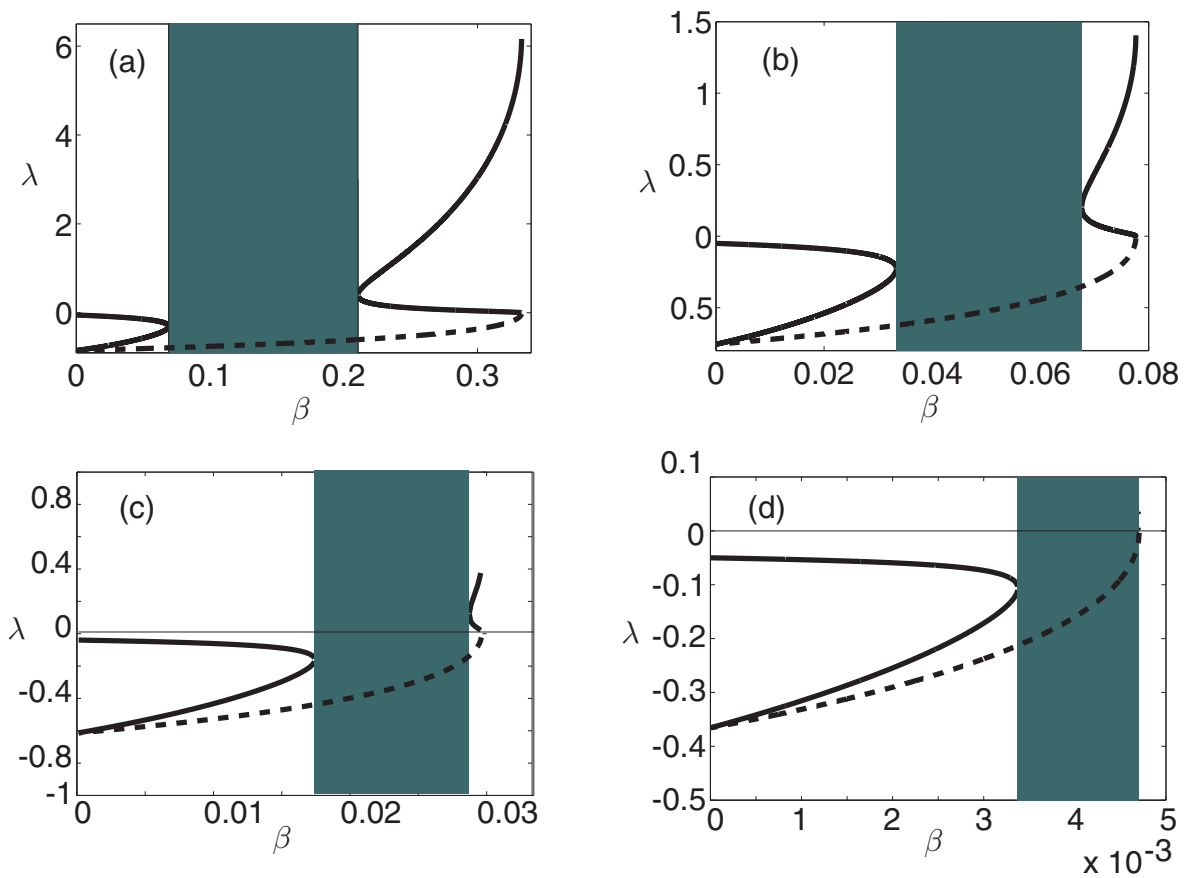

FIG. 3.3. Eigenvalues associated with radially symmetric perturbations of a stationary circular bump. Eigenvalues of the expansion (solid curves) and contraction (dashed curves) perturbations are plotted for (a) $\theta=0.01$, (b) $\theta=0.03$, (c) $\theta=0.05$, and (d) $\theta=0.07$. Stability analysis of expansion perturbations breaks down in the grey region due to the roots (3.30) being complex. Other parameters are $A=0.3, \sigma=4$, and $\alpha=20$.

which we verify to be true using (3.20) and (3.21). Therefore, we are able to show the existence of a zero eigenvalue, reflecting that a $2 \mathrm{D}$ stationary bump will be translationally invariant, as we would expect.

We now illustrate the stability properties of 2D bumps in the case of the Mexican hat weight function (3.7). We consider the upper branches of the existence curves shown in Figure 3.1, which in the given parameter regime are stable in the limit $\beta \rightarrow 0$. (As shown elsewhere [29], for a sufficiently small threshold $\theta$ such bumps would destabilize in the absence of synaptic depression, leading to the formation of stationary multibump solutions.) Since 2D bumps in neural fields with local negative feedback have previously been shown to give rise to moving spots - analogous to traveling pulses in one dimension - we expect that the dominant instability will be associated with a $D_{1}$-symmetric mode corresponding to a shift perturbation. Therefore, we will restrict ourselves to calculating the eigenvalues associated with low-order eigenmodes. In Figure 3.3, we plot the real eigenvalues corresponding to radially symmetric expansions and contractions as functions of $\beta$; see (3.30) and (3.32). As in the $1 \mathrm{D}$ case, the expansion mode dominates over contraction, due to more resources existing outside of the bump. Moreover, as $\beta$ increases, two negative roots of the characteristic equation (3.30) meet, and they become complex, violating our ansatz. When the eigenvalues become real again, they are both greater than zero, implying the bump will certainly be unstable. On the other hand, the real eigenvalue associated with contraction perturbations is always negative, approaching zero (and 
the lower positive branch of expansions) as the bump width approaches the value at which the upper and lower existence curves meet. As we show below (see Figure 3.4), the instability associated with positive real eigenvalues of the expansion perturbations typically occurs at larger values of $\beta$ than $D_{1}$-symmetric or shift perturbations. Moreover, in numerical simulations we were unable to find other types of instabilities, such as oscillatory ones, when systematically perturbing the bump with a wide range of perturbations; see section 4 . This suggests that Hopf bifurcations do not arise within the forbidden $\beta$ region of the expansion perturbations shown in Figure 3.3. Hence, shift perturbations tend to dominate radially symmetric bump instabilities.

Since we cannot derive an effective analytical method for computing nonradially symmetric eigensolutions of (3.23), we shall resort to numerically computing some of these solutions. Thus, we assume that each of the subdomains $\mathcal{A}_{+}$and $\mathcal{A}_{-}$is nonempty. We then develop a numerical approximation scheme to find solutions to the system of equations

$$
\begin{aligned}
(\lambda+1) \psi(a, \phi)-\Phi(a, \phi)= & a \gamma \int_{\mathcal{A}_{+}} \psi\left(a, \phi^{\prime}\right) w\left(2 a \sin \frac{\phi-\phi^{\prime}}{2}\right) \mathrm{d} \phi^{\prime} \\
& +\frac{a \gamma}{1+\alpha \beta} \int_{\mathcal{A}_{-}} \psi\left(a, \phi^{\prime}\right) w\left(2 a \sin \frac{\phi-\phi^{\prime}}{2}\right) \mathrm{d} \phi^{\prime}, \\
\left(\lambda+\alpha^{-1}+\beta\right) \Phi(a, \phi)= & -a \gamma \beta \int_{\mathcal{A}_{+}} \psi\left(a, \phi^{\prime}\right) w\left(2 a \sin \frac{\phi-\phi^{\prime}}{2}\right) \mathrm{d} \phi^{\prime} .
\end{aligned}
$$

We discretize this system by assigning $\phi_{j}:=(j-1) \Delta \phi, \psi_{j}:=\psi\left(a, \phi_{j}\right), \Phi_{j}:=\Phi\left(a, \phi_{j}\right)$, and $w_{j k}:=w\left(2 a \sin \frac{\phi_{j}-\phi_{k}}{2}\right)$, where $j=1, \ldots, N$ and $\Delta \phi=2 \pi / N$. Also, for a particular $n$-mode perturbation, discretized versions of each of the subdomains $\mathcal{A}_{+}$ and $\mathcal{A}_{-}$will be a collection of $n$ identically sized disconnected regions of $\phi_{j}$ values; we call these $A_{+}$and $A_{-}$, respectively. We call the $n$ identical disconnected regions of $A_{+}$and $A_{-}$lobes. To completely characterize the entirety of solutions for a particular value of $n$, we assume the number of grid points $l$ in each lobe of $A_{+}$is anywhere between 1 and $N / n-1$. Taking $l$ to be 0 or $N / n$ would imply a pure contraction or expansion perturbation, respectively. Thus, employing Riemann sums, a first-order accurate discretization of the system of equations (3.39) and (3.40) is

$$
\begin{aligned}
& \lambda \psi_{j}=-\psi_{j}+\Phi_{j}+a \gamma \Delta \phi \sum_{A_{+}} \psi_{k} w_{j k}+\frac{a \gamma \Delta \phi}{1+\alpha \beta} \sum_{A_{-}} \psi_{k} w_{j k}, \\
& \lambda \Phi_{j}=-\left(\alpha^{-1}+\beta\right) \Phi_{j}-a \gamma \beta \Delta \phi \sum_{A_{+}} \psi_{k} w_{j k},
\end{aligned}
$$

where $j=1, \ldots, N$. We now have a linear system, whose eigensolutions are approximations to the $n$-mode eigensolutions of (3.23). Thus, we can use an iterative method to solve for all of the eigensolutions of the system of equations (3.41) and (3.42). Although (3.41) and (3.42) will have many solutions, we find that only one or two will satisfy the original assumption regarding the number of grid points in the subdomains of $A_{+}$and $A_{-}$. With all possible eigensolutions in hand, we thus pick out those corresponding to perturbations of the $2 \mathrm{D}$ bump by employing the self-consistency conditions that $\psi_{j}>0$ when $j \in A_{+}$and $\psi_{j}<0$ when $j \in A_{-}$. For each of the $n$-mode perturbations that we have studied, a maximum of two different values of $l$ can occur (see Figure 3.5).

In Figure 3.4, we plot the real eigenvalues associated with some of the radially nonsymmetric perturbations (case (iii)) as functions of $\beta$. In particular, we plot 

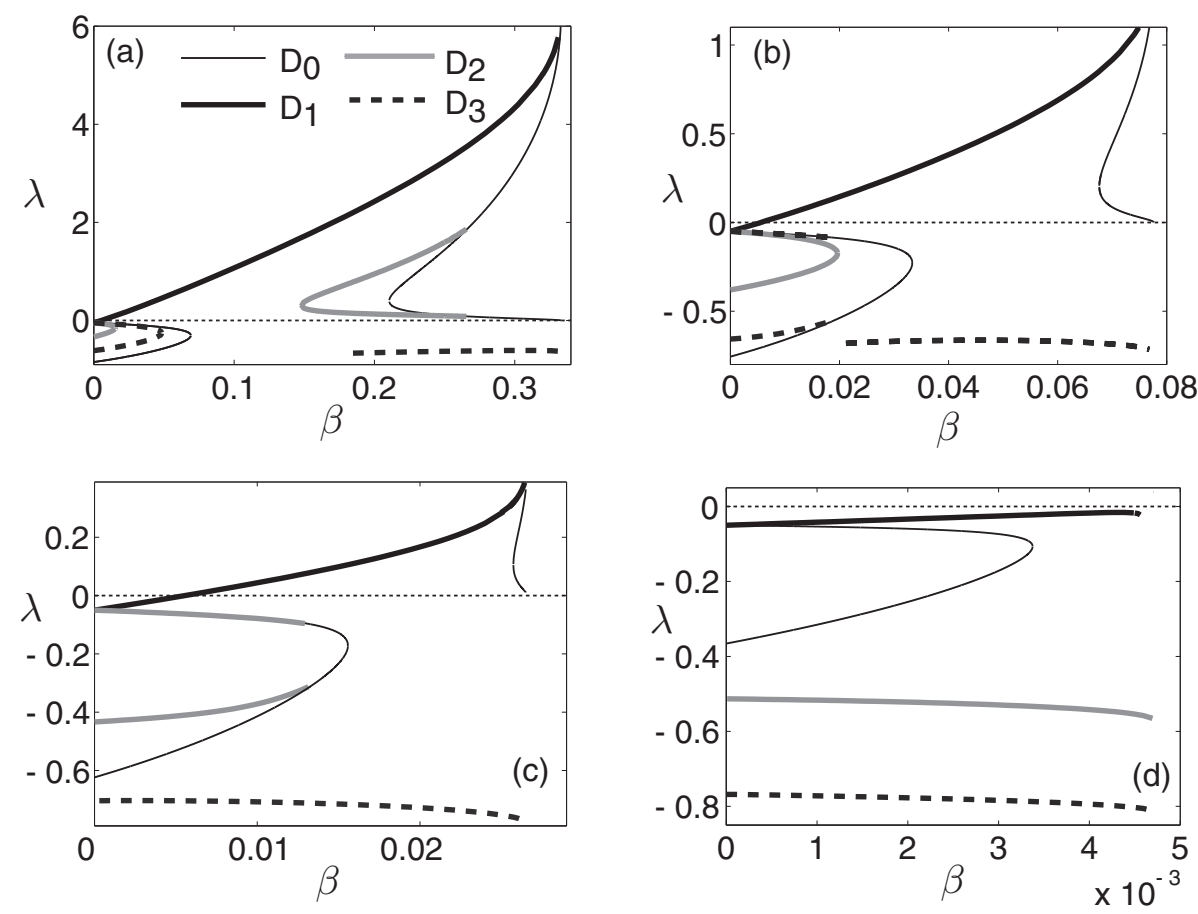

FIG. 3.4. Eigenvalues associated with radially nonsymmetric perturbations of a stationary circular bump. Eigenvalues of the $D_{1}$ (thick line), $D_{2}$ (grey line), and $D_{3}$ (dashed line) symmetric modes for (a) $\theta=0.01$, (b) $\theta=0.03$, (c) $\theta=0.05$, and (d) $\theta=0.07$. We also plot the eigenvalues of the radially symmetric expansion mode $D_{0}$ (thin line) from Figure 3.3. Other parameters are $A=0.3, \sigma=4$, and $\alpha=20$.

the nonzero $D_{1}, D_{2}$, and $D_{3}$ eigenvalues associated with perturbations having the respective symmetry. We operate in a region of parameter space where bumps are stable with respect to all $D_{n}$-symmetric perturbations as $\beta \rightarrow 0$ (see [29]). Here, by $D_{n}$-symmetry we mean that the solution that results from applying any scaling of the corresponding perturbation will have all of the $n$ rotational and reflectional symmetries of the dihedral group $D_{n}$. Figure 3.4 shows that there exists a nontrivial branch of $D_{1}$ eigenvalues (in addition to the zero eigenvalue arising from translation invariance) with $\lambda \rightarrow-\alpha^{-1}$ as $\beta \rightarrow 0$. As $\beta$ increases from zero, this $D_{1}$ branch can become positive (see Figure 3.4(a)-(c)), indicating instability of the bump with respect to shift perturbations. Analogous to the shift mode of the $1 \mathrm{D}$ system, which leads to traveling pulses, this $2 \mathrm{D}$ shift mode leads to a traveling spot when unstable; see section 4 . There also exist higher-order $\left(D_{n}, n=2,3\right)$ branches over particular intervals of $\beta$, but they all lie below the nontrivial $D_{1}$ branch, suggesting that the latter dominates any nonradially symmetric instabilities. In Figure $3.4(\mathrm{a})-(\mathrm{c})$ the positive $D_{1}$ branch also dominates the radially symmetric $\left(D_{0}\right)$ perturbations, whereas in Figure $3.4(\mathrm{~d})$ both the $D_{0}$ and $D_{1}$ branches remain negative. (More precisely, there does exist a pair of positive $D_{0}$ branches but only for an infinitesimally small range of $\beta$ values; see Figure 3.3(d).)

In Figures 3.5 and 3.6, we illustrate how the shape of nonsymmetric perturbations depends upon changes in $\beta$ for $\theta=0.01$ and $\theta=0.05$, respectively. Let $\left|\mathcal{A}_{n}\right|$ denote the arc length of each lobe of the domain $\mathcal{A}_{+}$for a given $n$. For pure Fourier modes 

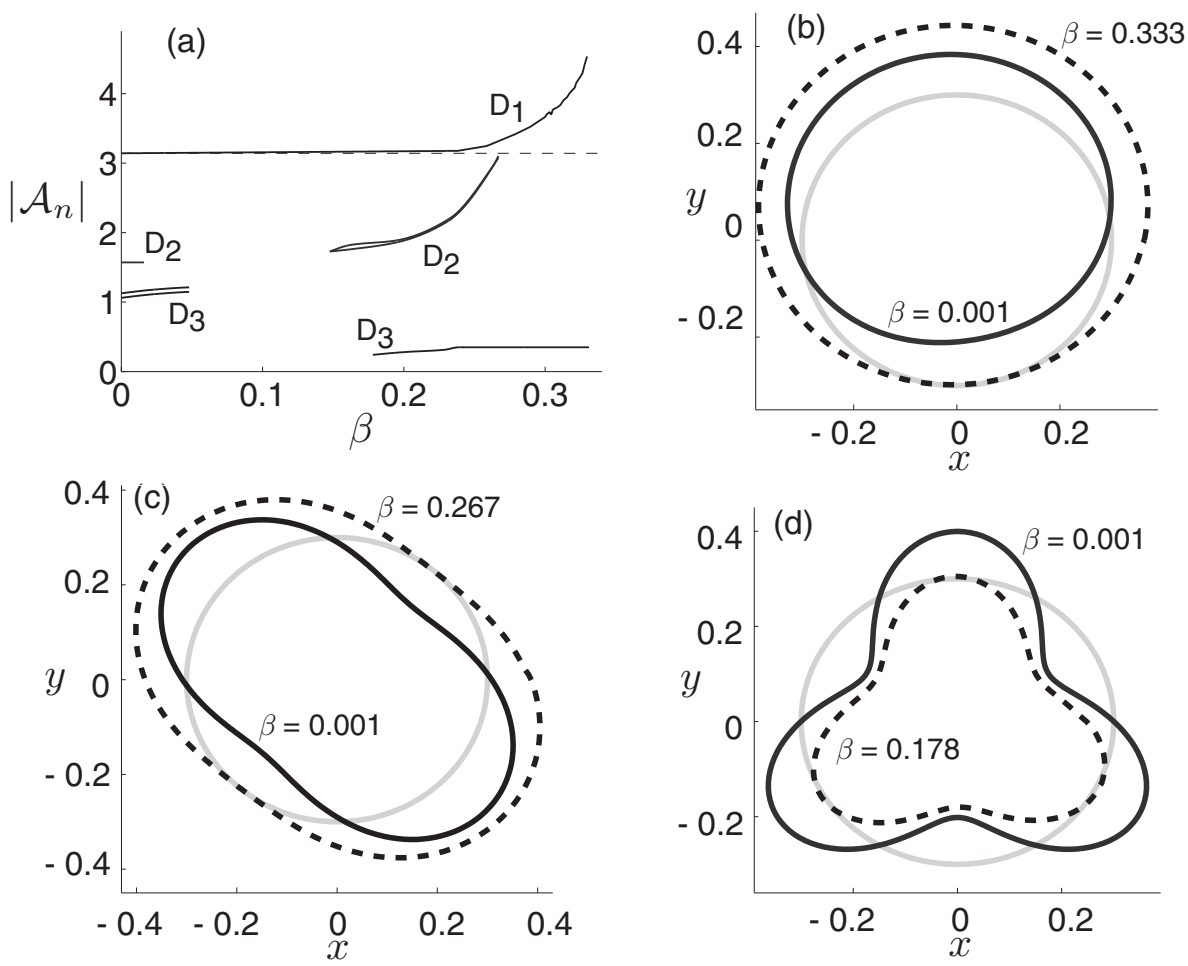

FIG. 3.5. The effect of $\beta$ on the shape of nonsymmetric eigenmodes for $\theta=0.01$. (a) Arc length $\left|\mathcal{A}_{n}\right|$ of each positive lobe of a $D_{n}$-symmetric eigenmode is plotted against $\beta$ for $n=1,2,3$. The thin dashed line represents $\pi$. Note that when more than one solution exists for a given $n$ and $\beta$, the sizes $\left|\mathcal{A}_{n}\right|$ of the lobes are almost identical. (b)-(d) Plot of the $D_{n}$-symmetric eigenmode with the maximal eigenvalue (in the case of multiple solutions) for different values of $\beta$ and $n=1,2,3$, respectively. In each case, the light grey circle represents the unperturbed radially symmetric bump solution. For sufficiently small $\beta$ (dark solid curves), the eigenmode is approximately a pure Fourier mode with $\left|\mathcal{A}_{n}\right| \approx \pi / n$. As $\left|\mathcal{A}_{n}\right|$ changes with increasing $\beta$, the eigenmodes become more distorted (dashed curves). Other parameters are $A=0.3, \sigma=4$, and $\alpha=20$. The stationary bump solution vanishes at the critical value $\beta_{c} \approx 0.333$.

$(\beta=0)$, we would have $\left|\mathcal{A}_{1}\right|=\pi,\left|\mathcal{A}_{2}\right|=\pi / 2$, and $\left|\mathcal{A}_{3}\right|=\pi / 3$. In Figure 3.5(a) and Figure 3.6(a) we plot $\left|\mathcal{A}_{n}\right|$ as a function of $\beta$ for $n=1,2,3$. In the case of the nontrivial $D_{1}$ eigenmode, $\left|\mathcal{A}_{1}\right|$ is a monotonically increasing function of $\beta$. As illustrated in Figures 3.5(b) and 3.6(b), the $D_{1}$ mode's positive lobes increase with $\beta$ such that $\left|\mathcal{A}_{1}\right| \rightarrow 2 \pi$ as $\beta \rightarrow \beta_{c}$, where $\beta_{c}$ is the saddle-point at which bumps cease to exist. In the case of $D_{2}$ eigenmodes, which typically exist in pairs, the arc length $\left|\mathcal{A}_{2}\right|$ is also an increasing function of $\beta$, but there are intervals over which $D_{2}$ eigenmodes do not exist. We find that $\left|\mathcal{A}_{2}\right| \rightarrow \pi$ as $\beta \rightarrow \beta_{2}<\beta_{c}$, where $\beta_{2}$ is the maximum value of $\beta$ for which $D_{2}$ eigenmodes exist. This is illustrated in Figures 3.5(c) and $3.6(\mathrm{c})$. Finally, we find at most two solutions with $D_{3}$ symmetry for a particular set of parameters. In Figure 3.5(a), the two initial $D_{3}$ branches have slightly different values of $\left|\mathcal{A}_{3}\right|$ prior to the solutions annihilating with one another at the critical value of $\beta \approx 0.042$. We find a sharp decrease in $\mathcal{A}_{3}$ when the two $D_{3}$ solution modes reappear at higher values of $\beta$. We plot the corresponding shape of the perturbation with a maximal eigenvalue in Figure 3.5(d) for a relatively small and large $\beta$ value. In Figure 3.6(a), we find only one $D_{3}$ solution branch whose lobe size $\left|\mathcal{A}_{3}\right|$ slightly 

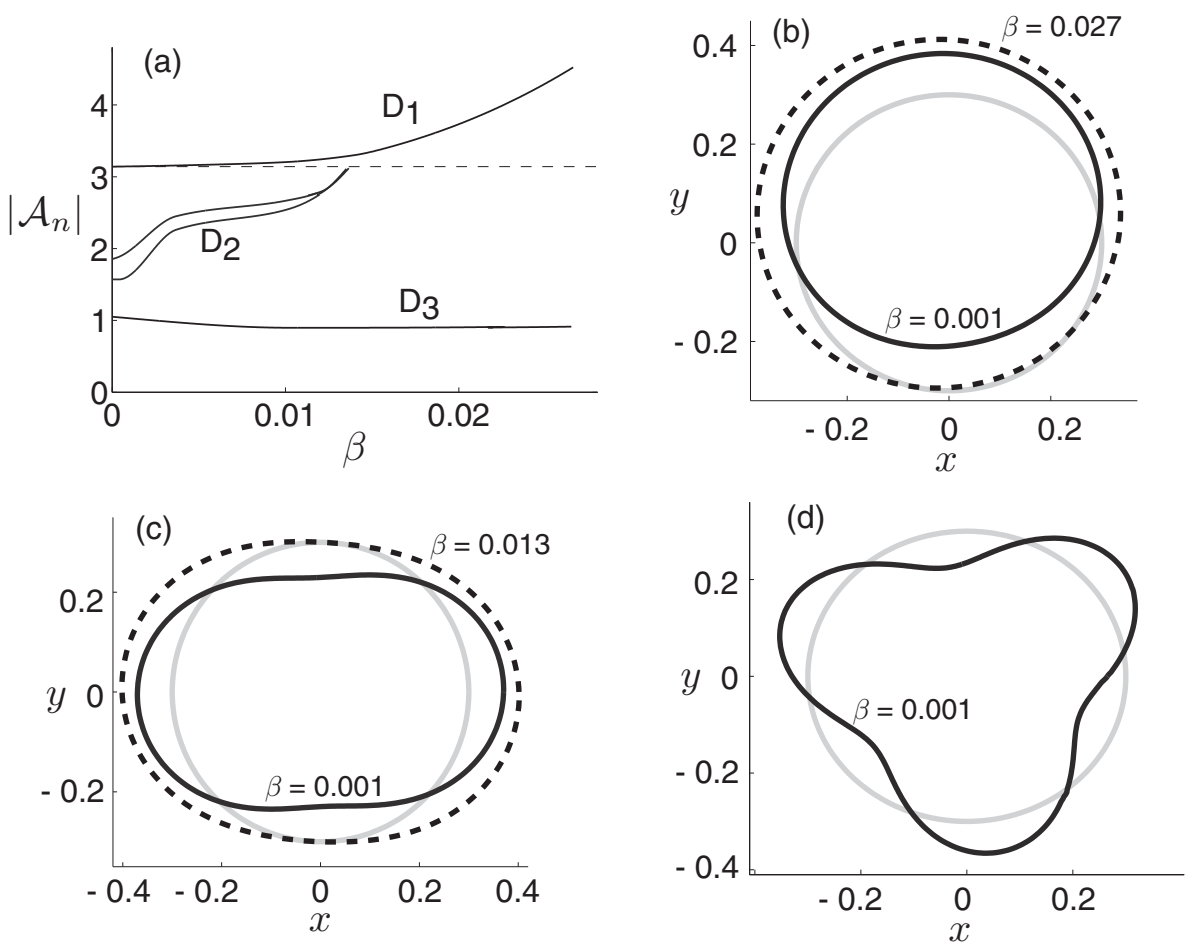

FIG. 3.6. Same as Figure 3.5 except that $\theta=0.05$. Here the $D_{3}$ eigenmode is only weakly dependent on $\beta$. The stationary bump solution vanishes at the critical value $\beta_{c} \approx 0.027$.

decreases as $\beta$ is increased. We plot the shape of the corresponding perturbation in Figure 3.6(d). As we alluded to in our discussion of the numerical methods, we find no more than two solutions, corresponding to two different values of $\left|\mathcal{A}_{n}\right|$, for each $n$-mode we have studied for a particular value of $\beta$.

4. Numerical simulations. We now study the full systems (1.6) and (3.1) using numerical approximation schemes. First, to evolve the 1D system (3.1) in time, we use a fourth-order Runge-Kutta method with 2000-4000 spatial grid points and a timestep of $d t=0.01$. The integral term in (1.6a) is approximated using Simpson's rule. We systematically examined whether taking finer grids changed stability results, and it does not. This is important because too coarse a grid can drastically alter numerical results, since discreteness can stabilize bumps that are not stable in the continuous system [16]. For all of our numerical simulations, we begin with an initial condition specified by an associated bump solution (2.5) that lies on the unstable part of the upper branch of the existence curves shown in Figure 2.1. After a brief period, we stimulate the system by adding an input perturbation of $u(x, t)$ defined as

$$
\psi_{ \pm}(x, t)=\chi(t)(w(x+a) \pm w(x-a)),
$$

which is motivated by eigenmodes of the linearized Amari equation (1.1). Leftward shifts (rightward shifts) correspond to $\psi_{-}(x, t)$ when $\chi(t) \geq 0(\chi(t) \leq 0)$, while expansions (contractions) correspond to $\psi_{+}(x, t)$ when $\chi(t) \geq 0(\chi(t) \leq 0)$. The resulting dynamics depends specifically on the type of perturbation applied to the bump. 

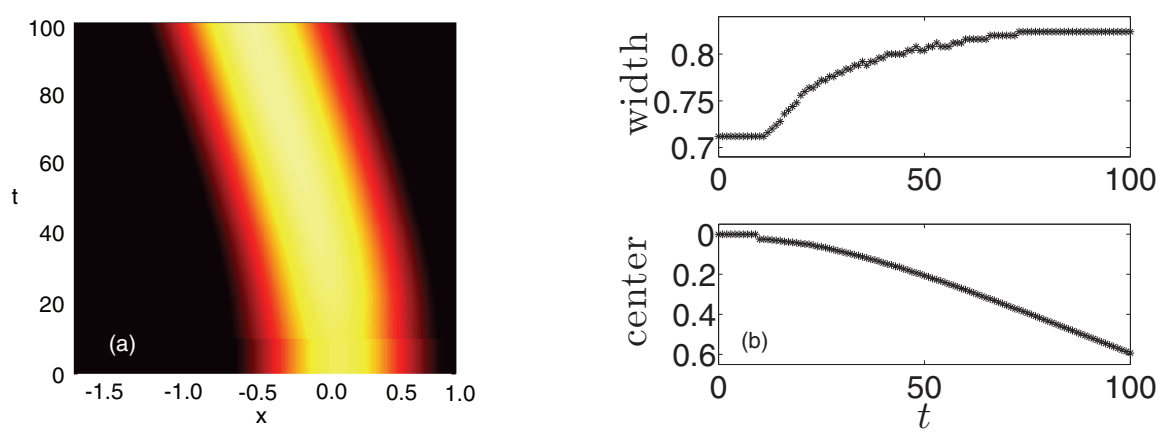

FIG. 4.1. Numerical simulation of a bump destabilized by a leftward shift perturbation. (a) Plot of $u(x, t)$ for an initial condition taken to be a stationary bump specified by (2.5). The solution is perturbed at $t=10$ by a leftward shift $\psi_{-}(x, t)$ such that $\chi(t)=-0.1$ for $t \in[10,10.1]$ and zero otherwise. (b) Bump width and center of mass plotted versus time. Bump width increases linearly following the perturbation, but eventually relaxes to a constant value as the solution evolves to a traveling pulse. The center of mass eventually moves linearly through space at the speed of the associated traveling pulse. Parameters are $A=0.6, \sigma=4, \alpha=20, \beta=0.009, \theta=0.1$.
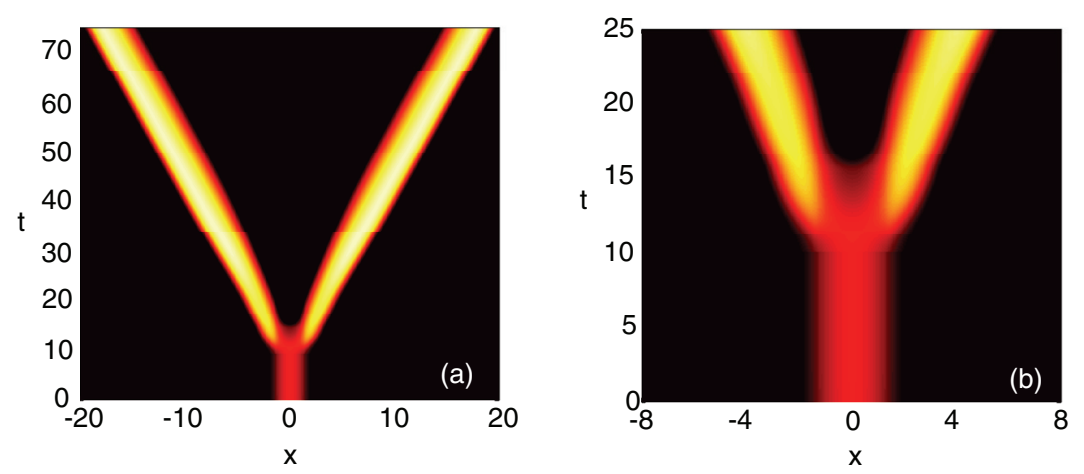

FIG. 4.2. Numerical simulation of a bump destabilized by an expanding perturbation. (a) Plot of $u(x, t)$ for an initial condition taken to be stationary bump specified by (2.5). The solution is perturbed at $t=10$ by an expansion $\psi_{+}(x, t)$ such that $\chi(t)=0.1$ for $t \in[10,10.1]$ and zero otherwise. (b) Plot of $u(x, t)$ for $t=0$ to $t=25$, showing initial expansion of the bump prior to splitting into two counterpropagating pulses. Parameters are $A=0.3, \sigma=4, \alpha=50, \beta=0.05$, $\theta=0.1$

When shift perturbations destabilize a bump, the resulting dynamics evolves to a traveling pulse solution. As we showed in previous work, synaptic depression is a reliable mechanism for generating traveling pulses in excitatory neural fields [19, 20]. As illustrated in Figure 4.1, following a perturbation by a leftward shift, the bump initially expands and then starts to propagate. Eventually, the traveling pulse's width stabilizes to a constant value, larger than the initial bump width. The initial linear growth in the bump's width is consistent with our linear stability calculations. In other simulations, we found that as synaptic depression strength $\beta$ is increased, the rate of linear growth in the width increases as well, which is also predicted by our stability analysis. In Figure 4.2, we show an example of how expansions destabilize the bump to result in two counterpropagating pulses. A closer look at the solution as a function of time immediately after the perturbation shows a transient phase, 
where the superthreshold region is still a connected domain, prior to the splitting into two pulses. As also predicted by our stability analysis, we found that contraction perturbations did not drive the system to the homogeneous zero state, unless their amplitude was large enough to drive the system to the other side of the separatrix given by the smaller unstable bump (see Figure 2.1).

Next, to evolve the 2D system (3.1) in time, we use a fourth-order Runge-Kutta method with a $200 \times 200$ spatial grid and a time-step of $d t=0.1$. The integral term in (3.1a) is approximated using Simpson's rule. We systematically examined whether taking finer grids changed stability results, and it does not. According to our stability analysis in the previous section, the dominant instability as $\beta$ is increased from zero is given by the shift perturbation. Thus, we explore this phenomenon at a point in parameter space where the bump is unstable to shifts (see Figure 3.4b). In Figure 4.3 , we show the results of a simulation in which the initial condition was taken to be a bump solution given by (3.9) with a shift perturbation added. We find the solution evolves to a traveling spot similar to those seen in a previous study of a $2 \mathrm{D}$ neural field with spike frequency adaptation [9]. The solution evolves to have an invariant profile as it travels along a path in space, roughly indicated by a grey line in Figure 3.5. This is analogous to traveling pulse solutions in one dimension generated due to bump instabilities in one dimension shown in Figures 4.1 and 4.2.

5. Discussion. In this paper we analyzed the existence and stability of $1 \mathrm{D}$ and 2D stationary bumps in a piecewise-smooth neural field model with synaptic depression. We showed that the local stability of a bump is determined by solutions to
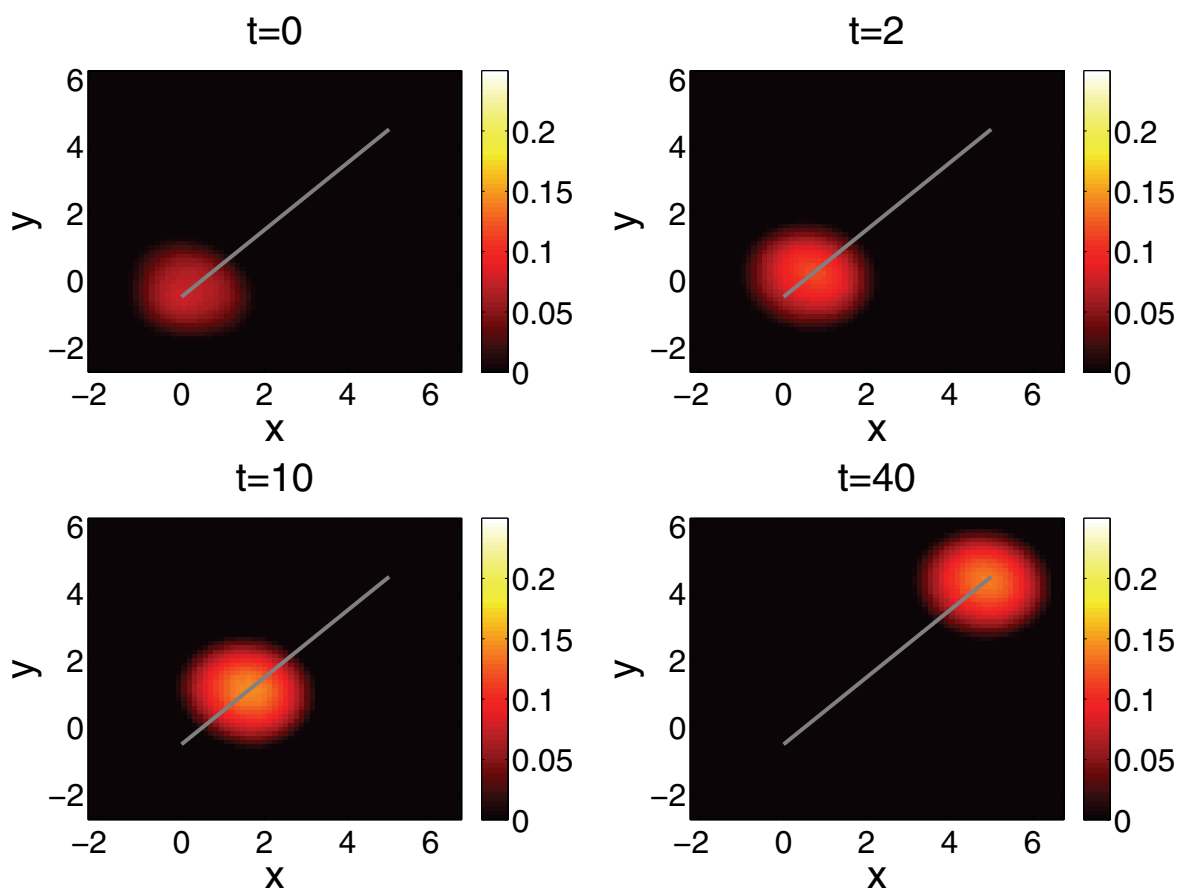

FIG. 4.3. Snapshots of a solution $u(x, y, t)$ to the integrodifferential equation (3.1) showing a bump destabilized at $t=0$ by a shift perturbation so that it evolves to a traveling spot. The grey line denotes the path through space. Parameters are $A=0.3, \sigma=4, \alpha=20, \beta=0.07, \theta=0.03$. 
a system of pseudolinear equations. For concreteness, we considered a Mexican hat weight function such that stable bumps exist in the limit $\beta \rightarrow 0$, where $\beta$ is the depletion rate of synaptic resources. As $\beta$ is increased from zero, the bump becomes unstable with respect to perturbations that shift the boundary of the bump, leading to the formation of a traveling pulse (1D) or a traveling spot (2D). One major effect of synaptic depression is that there is an asymmetry between the regions of contraction and expansion of the bump boundary. Physically, this can be understood from the fact that neural populations just outside of the stationary bump have maximal synaptic resources so they can recruit their nearest neighbors to continue spreading activity brought about by an initial expansion. On the other hand, neural populations within the interior of the bump do not possess the resources to continue the damping of activity via lateral inhibition brought about by an initial contraction.

There are various possible extensions of our work. First, our stability analysis assumed that the dominant instabilities were associated with nonoscillatory, separable solutions of pseudolinear equations such as (3.18) and (3.19). However, there are well-known scenarios in neural field models with linear adaptation, where Hopf bifurcations can occur leading to spatially structured oscillations such as breathers, target patterns, and spiral waves $[14,15,31,33,35]$. Recently we have shown how spatially structured oscillations can also occur in neural fields with synaptic depression, provided that the firing rate function has finite gain $[19,20]$. It would be interesting to explore scenarios where oscillations arise in neural fields with synaptic depression and Heaviside nonlinearities via some form of generalized Hopf bifurcation, along lines analogous to recent studies of nonsmooth dynamical systems [10, 44]. One recent example occurs in a competitive neural network model of binocular rivalry [22]. Second, piecewise-smooth neural field models arise for other types of nonlinear adaptation such as threshold dynamics, where negative feedback dynamically increases the threshold $\theta$ in response to an increased firing rate, corresponding to a form of spike frequency adaptation [8]. As we have recently shown, the treatment of the latter form of adaptation differs considerably from synaptic depression in the case of Heaviside firing rate functions [21]. Finally, just as synaptic depression is a direct form of local negative feedback, synaptic facilitation can provide an indirect feedback mechanism if it specifically amplifies inhibitory synapses $[36,45]$. Thus another extension is to consider a two population model, in which depression acts on excitatory synapses, while facilitation acts on inhibitory synapses; such a model is suggested by experimental studies of short term synaptic plasticity [28].

\section{REFERENCES}

[1] L. F. Aвbott, J. A. Varela, K. Sen, And S. B. Nelson, Synaptic depression and cortical gain control, Science, 275 (1997), pp. 220-224.

[2] S. Amari, Dynamics of pattern formation in lateral-inhibition type neural fields, Biol. Cybernet., 27 (1977), pp. 77-87.

[3] P. C. Bressloff, Spontaneous symmetry breaking in self-organizing neural fields, Biol. Cybernet., 93 (2005), pp. 256-274.

[4] P. C. Bressloff, Weakly interacting pulses in synaptically coupled excitable neural media, SIAM J. Appl. Math., 66 (2005), pp. 57-81.

[5] S Coombes, Waves, bumps and patterns in neural field theories, Biol. Cybernet., 93 (2005), pp. $91-108$.

[6] R. D. Chervin, P. A. Pierce, and B. W. Connors, Periodicity and directionality in the propagation of epileptiform discharges across neocortex, J. Neurophysiol., 60 (1988), pp. 1695-1713.

[7] S. Coombes And M. R. Owen, Evans functions for integral neural field equations with Heaviside firing rate function, SIAM J. Appl. Dyn. Syst., 3 (2004), pp. 574-600. 
[8] S. Coombes And M. R. Owen, Bumps, breathers, and waves in a neural network with spike frequency adaptation, Phys. Rev. Lett., 94 (2005), 148102.

[9] S. Coombes And M. R. Owen, Exotic dynamics in a firing rate model of neural tissue with threshold accommodation, in Fluids and Waves, Contemp. Math. 440, AMS, Providence, RI, 2007, pp. 123-144.

[10] M. Di Bernardo, C. J. Budd, A. R. Champneys, P. Kowalczyk, A. B. Nordmark, G. O. Tost, And P. T. PiIroinens, Bifurcations in nonsmooth dynamical systems, SIAM Rev., 50 (2008), pp. 629-701.

[11] G. B. ERmentrout, Neural networks as spatio-temporal pattern forming systems, Rep. Prog. Phys., 61 (1998), pp. 353-430.

[12] O. Faugeras, R. Veltz, and F. Grimbert, Persistent neural states: Stationary localized activity patterns in nonlinear continuous n-population, q-dimensional neural networks, Neural Comput., 21 (2009), pp. 147-187.

[13] R. FitzHugh, Impulses and physiological states in theoretical models of nerve membrane, Biophys. J., 1 (1961), pp. 445-466.

[14] S. E. Folias AND P. C. BRessloff, Breathing pulses in an excitatory neural network, SIAM J. Appl. Dyn. Syst., 3 (2004), pp. 378-407.

[15] S. E. Folias and P. C. Bressloff, Breathers in two-dimensional neural media, Phys. Rev. Lett., 95 (2005), 208107.

[16] Y. GuO AND C. C. CHOw, Existence and stability of standing pulses in neural networks: II. Stability, SIAM J. Appl. Dyn. Syst., 4 (2005), pp. 249-281.

[17] J. P. KeEner, Waves in excitable media, SIAM J. Appl. Math., 39 (1980), pp. 528-548.

[18] Z. P. Kilpatrick, S. E. Folias, And P. C. Bressloff, Traveling pulses and wave propagation failure in an inhomogeneous neural media, SIAM J. Appl. Dyn. Syst., 7 (2008), pp. 161185.

[19] Z. P. Kilpatrick and P. C. Bressloff, Effects of synaptic depression and adaptation on spatiotemporal dynamics of an excitatory neuronal network, Phys. D, 239 (2010), pp. 547560.

[20] Z. P. Kilpatrick And P. C. Bressloff, Spatially structured oscillations in a two-dimensional excitatory neuronal network with synaptic depression, J. Comput. Neurosci., 28 (2010), pp. 193-209.

[21] Z. P. Kilpatrick And P. C. Bressloff, Stability of bumps in piecewise smooth neural fields with nonlinear adaptation, Phys. D, 239 (2010), pp. 1048-1060.

[22] Z. P. Kilpatrick And P. C. Bressloff, Binocular rivalry in a competitive neural network with synaptic depression, SIAM J. Appl. Dyn. Syst., 9 (2010), pp. 1303-1347.

[23] K. Kishimoto and S. Amari, Existence and stability of local excitations in homogeneous neural fields, J. Math. Biol., 7 (1979), pp. 303-318.

[24] C. R. Laing, W. C. Troy, B. Gutkin, and G. B. Ermentrout, Multiple bumps in a neuronal model of working memory, SIAM J. Appl. Math., 63 (2002), pp. 62-97.

[25] C. R. Laing and W. C. Troy, PDE methods for nonlocal models, SIAM J. Appl. Dyn. Syst., 2 (2003), pp. 487-516.

[26] C. R. LAING, Spiral waves in nonlocal equations, SIAM J Appl. Dyn. Syst., 4 (2005), pp. $588-606$.

[27] J. S. Lund, A. Angelucci, And P. C. Bressloff, Anatomical substrates for functional columns in macaque monkey primary visual cortex, Cerebral Cortex, 12 (2003), pp. 15-24.

[28] O. Melamed, O. Barak, G. Silberberg, H. Markram, and M. Tsodyks, Slow oscillations in neural networks with facilitating synapses, J. Comput. Neurosci., 25 (2008), pp. 308-316.

[29] M. R. Owen, C. R. LAING, AND S. Coombes, Bumps and rings in a two-dimensional neural field: Splitting and rotational instabilities, New J. Phys., 9 (2007), 378. Available online at http://iopscience.iop.org/1367-2630/9/10/378.

[30] D. J. Pinto and G. B. Ermentrout, Spatially structured activity in synaptically coupled neuronal networks: I. Traveling fronts and pulses, SIAM J. Appl. Math., 62 (2001), pp. $206-225$.

[31] D. J. Pinto And G. B. Ermentrout, Spatially structured activity in synaptically coupled neuronal networks: II. Lateral inhibition and standing pulses, SIAM J. Appl. Math., 62 (2001), pp. 226-243.

[32] B. SANDSTEDE, Evans functions and nonlinear stability of traveling waves in neuronal network models, Internat. J. Bifur. Chaos, in Appl. Sci. Engrg., 17 (2007), pp. 2693-2704.

[33] V. Shusterman and W. C. Troy, From baseline to epileptiform activity: A path to synchronized rhythmicity in large-scale neural networks, Phys. Rev. E, 77 (2008), 061911.

[34] J. Tabak, W. Senn, M. J. O'Donovan, and J. Rinzel, Modeling of spontaneous activity in developing spinal cord using activity-dependent depression in an excitatory network, J. Neurosci., 20 (2000), pp. 3041-3056. 
[35] W. C. Troy and V. Shusterman, Patterns and features of families of traveling waves in large-scale neuronal networks, SIAM J. Appl. Dyn. Syst., 6 (2007), pp. 263-292.

[36] M. V. Tsodyks And H. Markram, The neural code between neocortical pyramidal neurons depends on neurotransmitter release probability, Proc. Natl. Acad. Sci. USA, 94 (1997), pp. $719-723$.

[37] M. Tsodyks, K. Pawelzik, And H. Markram, Neural networks with dynamic synapses, Neural Comput., 10 (1998), pp. 821-835.

[38] X.-J. WANG, Synaptic reverberation underlying mnemonic persistent activity, Trends Neurosci., 24 (2001), pp. 455-463.

[39] H. WERNER AND T. RiChteR, Circular stationary solutions in two-dimensional neural fields, Biol. Cybern., 85 (2001), pp. 211-217.

[40] H. R. Wilson AND J. D. CowAn, A mathematical theory of the functional dynamics of cortical and thalamic nervous tissue, Kybernetik, 13 (1973), pp. 55-80.

[41] J.-Y. Wu, L. Guan, And Y. Tsau, Propagating activation during oscillations and evoked responses in neocortical slices, J. Neurosci., 19 (1999), pp. 5005-5015.

[42] J.-Y. Wu, Propagating waves of activity in the neocortex: What they are, what they do, Neuroscientist, 14 (2008), pp. 487-502.

[43] L. ZHANG, Traveling waves of singularly perturbed system of integral-differential equations arising from neuronal networks, J. Dynam. Differential Equations, 17 (2005), pp. 489-522.

[44] Y. Zou, T. Kupper, AND W. J. Beyn, Generalized Hopf bifurcation for planar Filippov systems continuous at the origin, J. Nonlinear Sci., 16 (2006), pp. 159-177.

[45] R. S. Zucker and W. G. Regehr, Short-term synaptic plasticity, Ann. Rev. Physiol., 64 (2002), pp. 355-405. 